\title{
ARTICLE
}

\section{Identification of the elementary structural units of the DNA damage response}

Francesco Natale ${ }^{1, \star}$, Alexander Rapp ${ }^{1, \star}$, Wei Yu ${ }^{1, \dagger}$, Andreas Maiser ${ }^{2}$, Hartmann Harz ${ }^{2}$, Annina Scholl ${ }^{1}$, Stephan Grulich", Tobias Anton ${ }^{2}$, David Hörl ${ }^{2}$, Wei Chen ${ }^{3}$, Marco Durante ${ }^{4, \dagger}$, Gisela Taucher-Scholz ${ }^{4}$, Heinrich Leonhardt ${ }^{2} \&$ M. Cristina Cardoso ${ }^{1}$

Histone $\mathrm{H} 2 \mathrm{AX}$ phosphorylation is an early signalling event triggered by DNA double-strand breaks (DSBs). To elucidate the elementary units of phospho-H2AX-labelled chromatin, we integrate super-resolution microscopy of phospho-H2AX during DNA repair in human cells with genome-wide sequencing analyses. Here we identify phospho-H2AX chromatin domains in the nanometre range with median length of $\sim 75 \mathrm{~kb}$. Correlation analysis with over 60 genomic features shows a time-dependent euchromatin-to-heterochromatin repair trend. After X-ray or CRISPR-Cas9-mediated DSBs, phospho-H2AX-labelled heterochromatin exhibits DNA decondensation while retaining heterochromatic histone marks, indicating that chromatin structural and molecular determinants are uncoupled during repair. The phosphoH2AX nano-domains arrange into higher-order clustered structures of discontinuously phosphorylated chromatin, flanked by CTCF. CTCF knockdown impairs spreading of the phosphorylation throughout the 3D-looped nano-domains. Co-staining of phospho-H2AX with phospho-Ku70 and TUNEL reveals that clusters rather than nano-foci represent single DSBs. Hence, each chromatin loop is a nano-focus, whose clusters correspond to previously known phospho-H2AX foci.

\footnotetext{
${ }^{1}$ Department of Biology, Technische Universität Darmstadt, 64287 Darmstadt, Germany. ${ }^{2}$ Department of Biology II, Center for Integrated Protein Science Munich (CIPSM), LMU Munich, 82152 Planegg-Martinsried, Germany. ${ }^{3}$ Max Delbrück Center for Molecular Medicine, 13125 Berlin, Germany. ${ }^{4}$ Department of Biophysics, GSI Helmholtzzentrum für Schwerionenforschung, 64291 Darmstadt, Germany. * These authors contributed equally to this work. $\dagger$ †resent addresses: G5 Lymphocyte Development and Oncogenesis, Immunology Department, Pasteur Institute, 75724 Paris Cedex 15, France (W.Y.); Trento Institute for Fundamental Physics and Application (TIFPA-INFN), via Sommarive 14, 38123 Trento, Italy (M.D.). Correspondence and requests for materials should be addressed to M.C.C. (email: cardoso@bio.tu-darmstadt.de).
} 
$\mathrm{D}$ NA double-strand breaks (DSBs) are the most harmful lesions induced by either endogenous (for example, replication) or exogenous (for example, ionizing radiation-IR) genotoxic stress, which may lead to chromosomal aberrations and tumorigenesis if not correctly repaired. To deal with DSBs, cells activate a rapid and hierarchically coordinated signalling cascade known as DNA damage response (DDR), leading to cell cycle arrest and allowing the DNA repair machinery to exert its function. One of the earliest events of DDR is the phosphatidylinositol-3-kinase-like-dependent phosphorylation of serine 139 of histone $\mathrm{H} 2 \mathrm{AX}(\gamma \mathrm{H} 2 \mathrm{AX})^{1}$, a histone $\mathrm{H} 2 \mathrm{~A}$ variant whose role at the interface of DNA repair, chromatin structure regulation and cell cycle checkpoint activation $^{2}$ is yet to be fully elucidated.

Detection of $\gamma \mathrm{H} 2 \mathrm{AX}$ has become the most widely used method for quantification of DSBs and their repair kinetics. Activated DDR, as scored by quantification of nuclear $\gamma \mathrm{H} 2 \mathrm{AX}$ focal structures, has been extensively described in both precancerous and cancer cells $s^{3,4}$. The majority of these studies were performed by conventional microscopy techniques, including confocal microscopy, and the structures resolved were in the micrometre or sub-micrometre range, with a predicted DNA content in the megabase-pair (Mbp) range. Indeed, $\gamma \mathrm{H} 2 \mathrm{AX}$ is proposed to spread up to several Mbps from the original lesion site, in higher eukaryotes $^{5}$. The distribution of such histone modification is neither symmetrical around DSB sites nor uniform on chromatin, as assessed by chromatin immunoprecipitation (ChIP) studies conducted in mammals ${ }^{6-8}$ and yeast ${ }^{9,10}$. Such uneven spreading may be accounted for by gene transcription ${ }^{11}$, or cohesin complex binding $^{12}$, which antagonize $\gamma \mathrm{H} 2 \mathrm{AX}$ formation along the chromosomes.

An increasing body of evidence underlines the crucial role of genome topology and chromatin spatial organization in the regulation of biological processes ${ }^{13}$. Recent chromosome conformation capture studies have revealed the complexity of genome architecture, with large compartments in the Mbp range conserved across cell lineages and species ${ }^{14,15}$, as well as smaller contact domains with a variable size in the range of a few hundreds of kilobase pairs $(\mathrm{kb})^{15}$. This spatial organization can be dynamic and underlines cell-type-specific networks, possibly driving the expression of specific sets of genes ${ }^{16}$ or organizing the replication process ${ }^{17}$

Nonetheless, the three-dimensional (3D) arrangement of $\gamma \mathrm{H} 2 \mathrm{AX}$-decorated chromatin in the nuclear volume and its dynamic evolution during the DDR remains elusive. Here we investigate the DDR over time at nanometre resolution by employing super-resolution microscopy techniques on human cells exposed to X-ray radiation. By overcoming the optical diffraction limit, structured illumination microscopy (3D-SIM) $)^{18}$ and stimulated-emission-depletion (STED) ${ }^{19}$ fluorescence microscopy present high prospecting capacity, thus allowing us to dissect complex structures of $\gamma \mathrm{H} 2 \mathrm{AX}$-decorated chromatin at nanometre resolution $(\sim 100 \mathrm{~nm})$. Furthermore, the integration of the microscopy results with CRISPR-Cas-targeted DNA damage, RNAi of the key structural factor CCCTC-binding factor (CTCF), $\gamma \mathrm{H} 2 \mathrm{AX}$ ChIP-Seq(uencing) profiles during DDR, and more than 60 genomic features reveal temporal, functional and structural insights into the elementary chromatin units read by the DNA DSB repair machinery.

\section{Results}

Cellular system and experimental strategy validation. For our study, we employed HeLa cells, an established human cell line whose (epi)genome is extensively annotated in the context of the ENCODE project (genome.ucsc.edu/ENCODE/). To test the DDR, we assessed the formation of $\gamma \mathrm{H} 2 \mathrm{AX}$ before and after exposure to IR. We investigated the early $(0.5 \mathrm{~h})$, mid $(3 \mathrm{~h})$ and late $(24 \mathrm{~h})$ stages of DDR, which, according to earlier reports ${ }^{20}$, represent $60-100 \%, 20-60 \%$ and less than $10 \%$ of the initial DSBs, respectively. Our confocal immunofluorescence analysis of $\gamma \mathrm{H} 2 \mathrm{AX}$ revealed that the show endogenous $\gamma \mathrm{H} 2 \mathrm{AX}$ signal. This is frequently observed in cancer cell lines and can be attributed to randomly produced DSBs at stalled and collapsed replication forks ${ }^{21,22}$. On exposure to IR, $\gamma \mathrm{H} 2 \mathrm{AX}$ followed the predicted repair kinetics, with nuclear $\gamma \mathrm{H} 2 \mathrm{AX}$ fluorescence intensity increasing, and then decreasing over time (Supplementary Fig. 1A). Similar kinetics was observed by western blot analysis (Supplementary Fig. 1B). Together, these methods revealed a four- to eightfold increase in $\gamma \mathrm{H} 2 \mathrm{AX}$ signal after IR. Overall, cells were able to activate a DDR and underwent cell cycle arrest, accumulating in S-phase (Supplementary Fig. 1C). No apoptosis was detected (Supplementary Fig. 1D), and $24 \mathrm{~h}$ post IR cells were viable, re-entered the cell cycle (Supplementary Fig. 1C) and proliferated, although at a lower rate compared with the mockirradiated controls (Supplementary Fig. 1E).

To investigate $\gamma \mathrm{H} 2 \mathrm{AX}$ kinetics at high resolution, we recorded super-resolution image sets before and during DDR, and acquired $\gamma \mathrm{H} 2 \mathrm{AX}$ ChIP-Seq genome-wide data at matching time points (Fig. 1a). In all of our immuno-based approaches, we probed $\gamma \mathrm{H} 2 \mathrm{AX}$-decorated chromatin with the same antibody, whose specificity was verified by slot blot analysis employing the $\gamma \mathrm{H} 2 \mathrm{AX}$-immunizing peptide (Supplementary Fig. 1F). The reproducibility of the sequencing data was assessed and confirmed by comparing biological replicates (Supplementary Fig. 1G).

Super-resolution microscopy of $\gamma \mathrm{H} 2 \mathrm{AX}$ kinetics during DDR. To first address the effect of improved optical resolution, we compared the number of $\gamma \mathrm{H} 2 \mathrm{AX}$ foci from cells imaged by conventional confocal and 3D-SIM microscopy, and analysed in addition the pseudo-wide-field images re-computed from the same 3D-SIM images, before and after deconvolution (Fig. 1b). A detailed analysis workflow is in the 'Methods' section and summarized in Supplementary Fig. 1H. Compared with confocal images (Fig. 1c), we observed a fivefold increase in foci numbers in pseudo-wide-field images, with an additional twofold increase in deconvolved images (Fig. 1d). Despite employing IR doses that are challenging for conventional confocal microscopy (10 Gy $\mathrm{X}$-ray), the enhanced optical resolution enabled us to resolve thousands of foci, increasing by about one order of magnitude the foci counts compared with the pseudo-wide-field, and about two orders of magnitude when comparing with confocal microscopy (Fig. 1e). Thus, it becomes obvious that a single focus identified by confocal microscopy can be further resolved by 3D-SIM into substructures (Fig. 1b, bottom panels, and Supplementary Fig. 2A), which we referred to as nano-foci. In addition, we controlled the imaging and reconstruction process of 3D-SIM by visual inspection of the reconstructed images in Fourier's space (Supplementary Fig. 2B). No reconstruction artifacts are visible as can be seen from the fast Fourier transformed images, which would contain regular stripe patterns otherwise.

Coherently, we observed a two- to fourfold decrease in the diameters of the segmented objects, when comparing 3D-SIM images with re-computed pseudo-wide-field images, with or without deconvolution, respectively (Supplementary Fig. 3A). Notably, in the 3D-SIM images, the nano-foci diameters were constant during the DDR (median lateral diameter: $\sim 200 \mathrm{~nm}$; Fig. 2a), indicating that we detected the smallest substructures of $\gamma \mathrm{H} 2 \mathrm{AX}$-decorated chromatin at the limit defined by the foci segmentation process (eight voxels). To gauge the actual size of $\gamma \mathrm{H} 2 \mathrm{AX}$ nano-foci, we recorded $\gamma \mathrm{H} 2 \mathrm{AX}$ immunofluorescence 
a

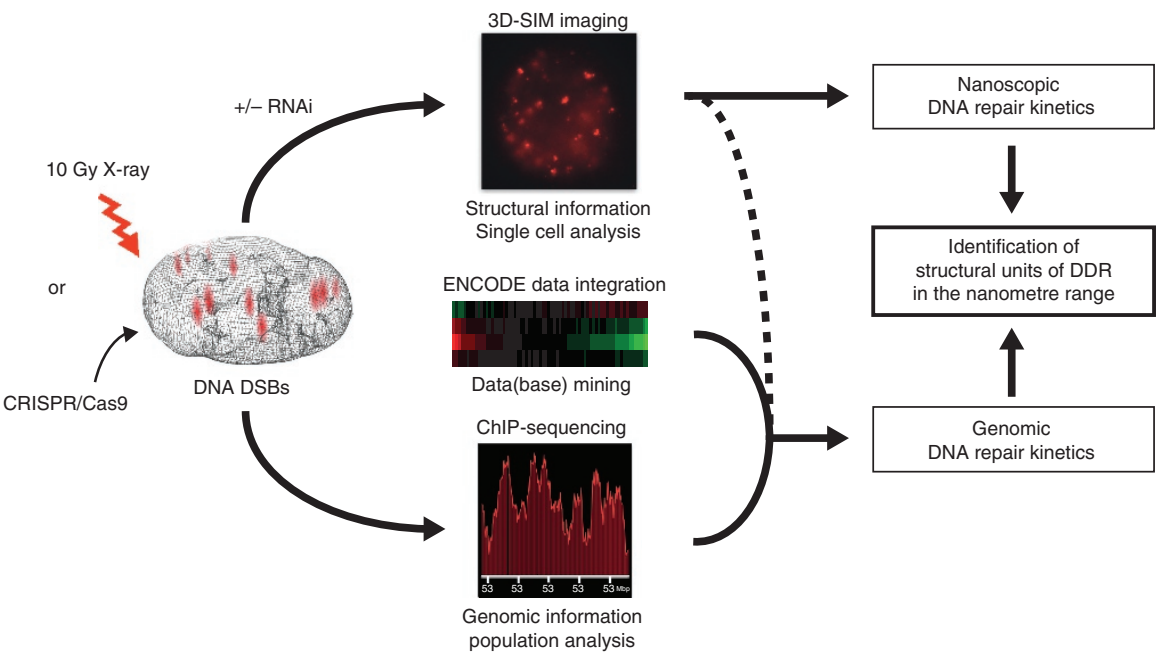

b
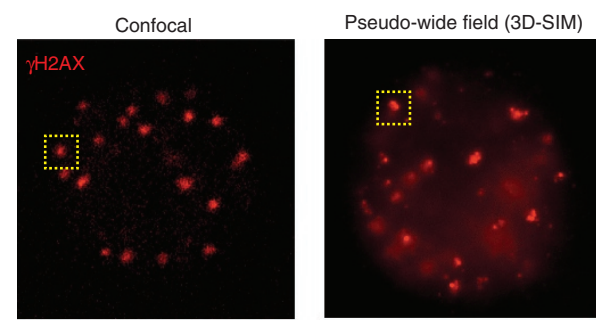

+ Deconvolution (3D-SIM)

3D-SIM
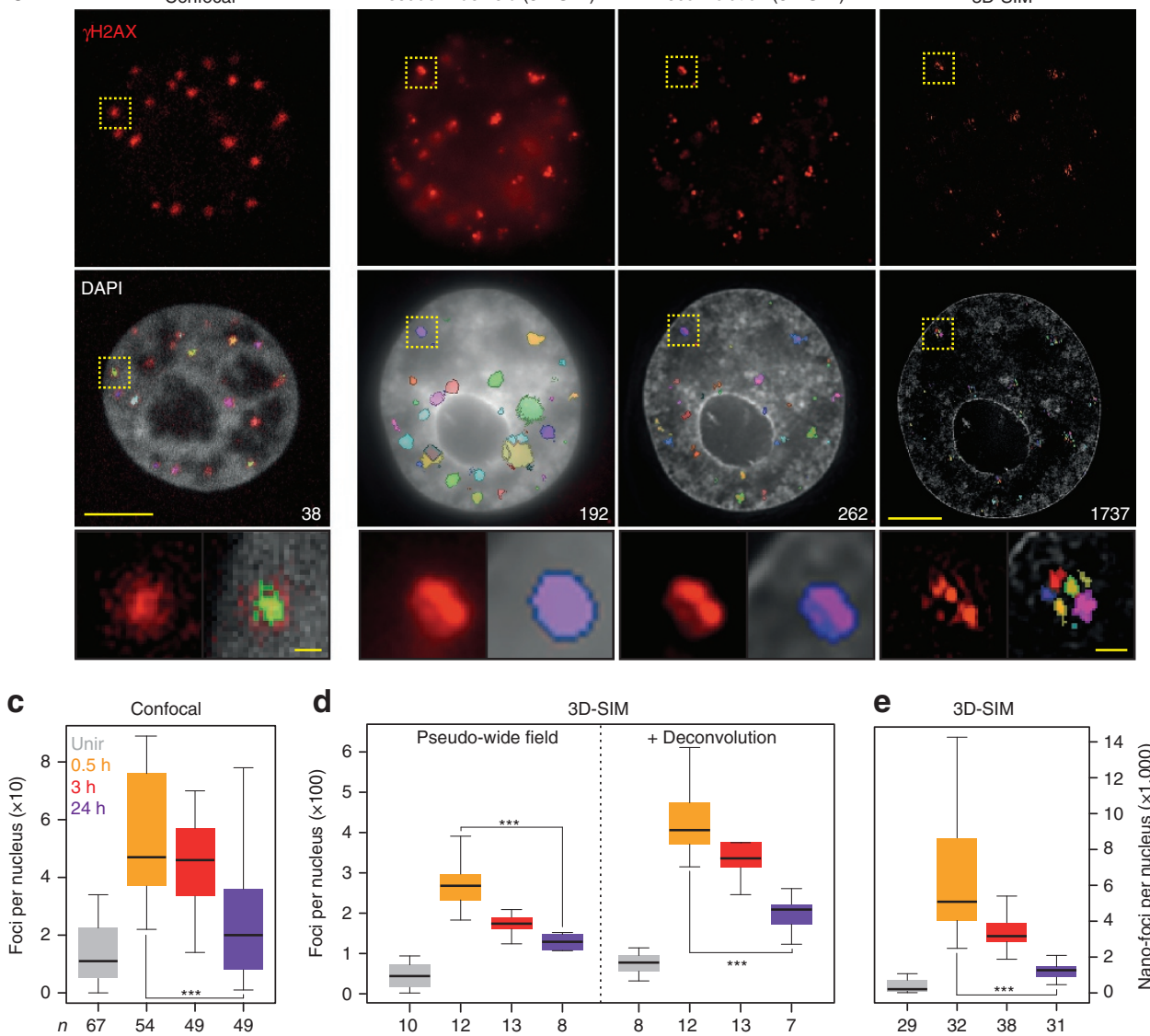

\begin{abstract}
d
\end{abstract}
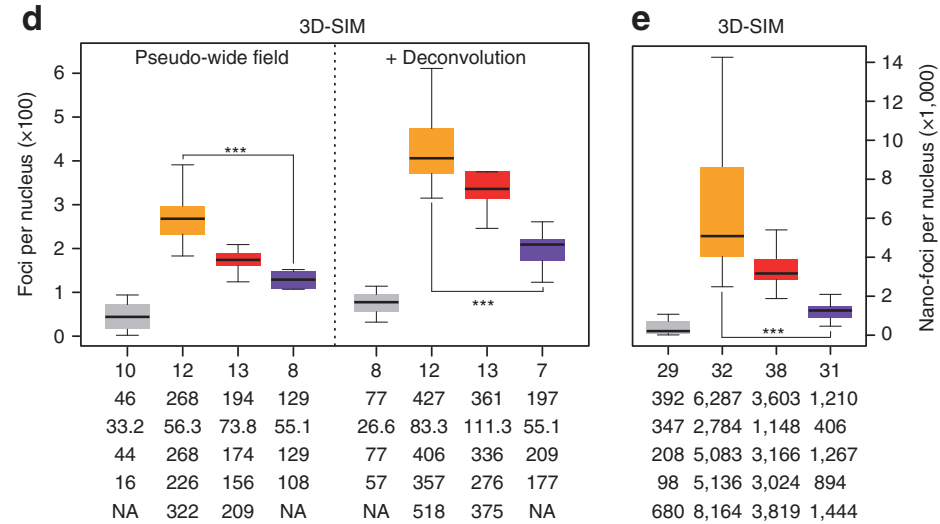

Figure 1 | Characterization of $\gamma \mathbf{H 2 A X}$ foci at different resolution levels. (a) Schematics of the experimental approach. (b) Mid-nuclear sections of confocal microscopy (z: $200 \mathrm{~nm}$ ) and 3D-SIM (z: $125 \mathrm{~nm}$ ) representative images of cells, $24 \mathrm{~h}$ post IR. Only for 3D-SIM, the same exemplary cell is shown as re-computed pseudo-wide-field image before or after deconvolution as well as the original 3D-SIM output. The total number of detected foci (highlighted in colours) in the whole nuclear volume is shown in the DAPI panels. The lower panels show magnified views of the yellow dashed frame. Scale bars, $5 \mu \mathrm{m}$ and $500 \mathrm{~nm}$ for main micrographs and magnified regions, respectively. $\gamma \mathrm{H} 2 \mathrm{AX}$ foci number distributions before and during DDR, from confocal images (c), 3D-SIM re-computed pseudo-wide-field of identical cell nuclei, before or after deconvolution (d) and original 3D-SIM images (e). $n$ : total number of imaged cells from three independent experiments. All boxes and whiskers represent 25-75 percentiles and three times the IQD. The mean number of foci and corresponding s.d., the median as well as the $95 \%$ confidence intervals $(\mathrm{Cl})$ for the median are shown below each box. NA: not applicable. For c-e: one-way ANOVA with Dunnett's correction; ${ }^{\star \star \star} P<10^{-3}$. 
a
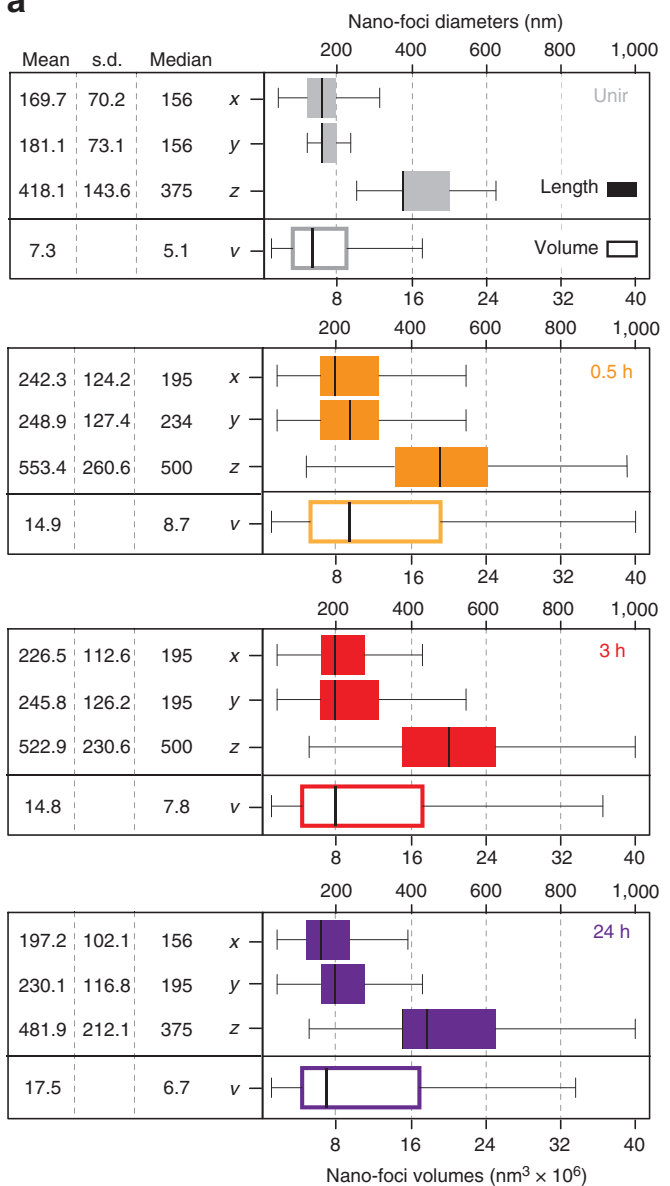

b

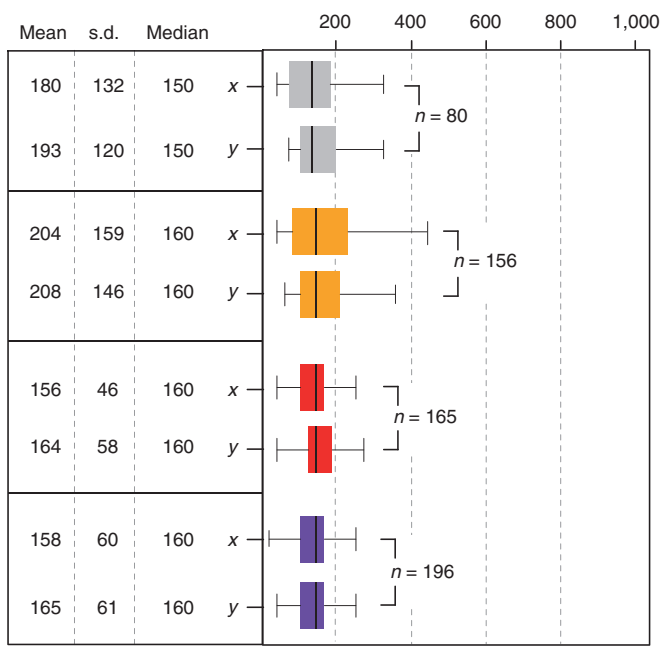

C

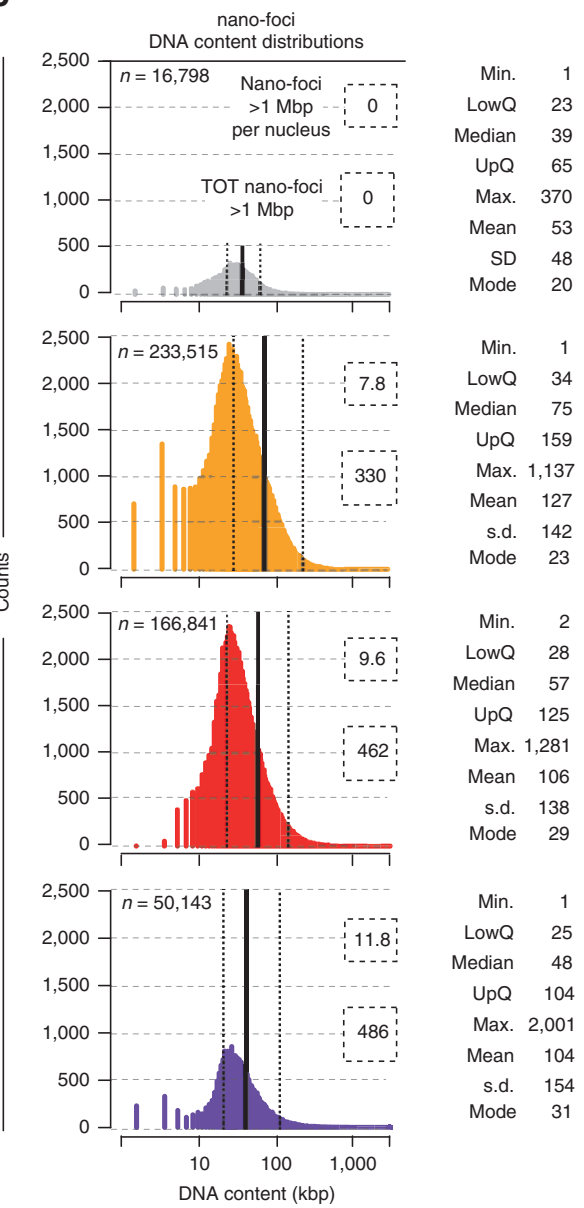

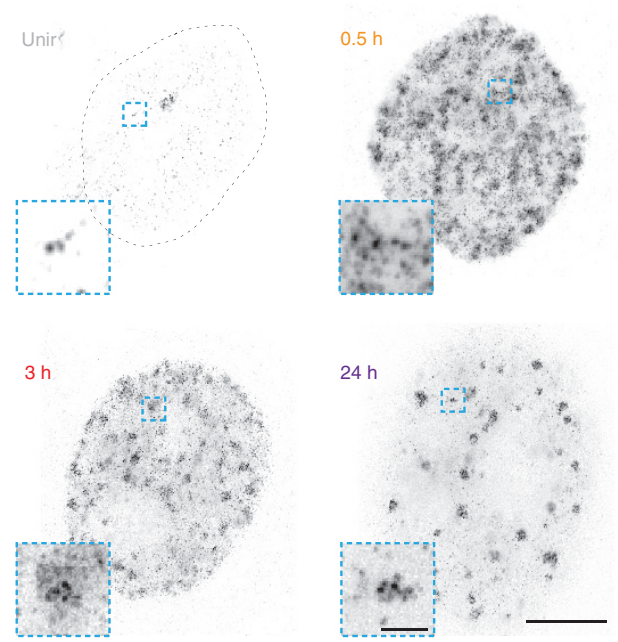

Figure 2 | Metrics of $\gamma \mathbf{H}$ 2AX nano-foci dimensions and DNA content. (a) Quantification of nano-foci diameters in the three dimensions (filled boxes, top) during DDR. From these three dimensions, the volumes were calculated (empty boxes, bottom). The difference between lateral and axial measurements is due to the lower resolution in the axial direction. Figures in $\mathrm{nm}$ or $\mathrm{nm}^{3} \times 10^{6}$ are shown. (b) STED microscopy of $\gamma \mathrm{H} 2 \mathrm{AX}$ immunofluorescence. (left) Quantification of lateral diameters of $\gamma \mathrm{H} 2 \mathrm{AX}$ nano-foci. Statistics and size scale are as in a. (right) Exemplary STED images of cells before and after IR are shown together with the magnified views of the light-blue boxes. Scale bars, $5 \mu \mathrm{m}$ and $500 \mathrm{~nm}$ for main micrographs and magnified regions, respectively. (c) DNA content distributions of $\gamma \mathrm{H} 2 \mathrm{AX}$ nano-foci before and during DDR. Only in IR-exposed cells, we found nano-foci larger than $1 \mathrm{Mbp}$ (dashed boxes), and their frequency never exceeded $1 \%(0.14 \%, 0.28 \%, 0.95 \%$ for $0.5 \mathrm{~h}, 3 \mathrm{~h}$ and $24 \mathrm{~h}$, respectively $)$. Kruskal-Wallis $\chi^{2}=18,503, \mathrm{df}=3, P<2.2 \times 10^{-16}$. Statistics (in kb) are shown next to each distribution. All boxes and whiskers are as in Fig. 1. $n$ : total number of measured nano-foci from all imaged cells in two independent experiments, for 3D-SIM (a,c) or STED (b). 
images by STED microscopy. Compared with our 3D-SIM set-up, STED provided a twofold increase in optical resolution ${ }^{18}$. Yet, the measured lateral diameters (Fig. 2b) were statistically undistinguishable from those recorded by 3D-SIM under sham-irradiation conditions (unpaired two-tailed $t$-test: $P>0.05$ ). Upon irradiation, the mean lateral diameters imaged by STED were only $\sim 20 \%$ smaller than those we measured by 3D-SIM imaging (unpaired two-tailed $t$-test: $P<10^{-3}$ ). These results validate our $3 \mathrm{D}$-SIM measurements and indicate that $\gamma \mathrm{H} 2 \mathrm{AX}$ nano-foci are the chromatin elementary units of the cellular response to DSBs.

Next, to estimate the DNA content of nano-foci, we related the integrated 4,6-diamidino-2-phenylindole (DAPI) intensity of each $\gamma \mathrm{H} 2 \mathrm{AX}$ nano-focus to the total DNA content represented by the integrated whole nuclear DAPI intensity (Supplementary Fig. 3B). The resulting DNA fractions were first corrected for the total HeLa genome size (determined by spectral karyotyping, Supplementary Fig. 3C), and then further corrected for the cell cycle phase of each given cell (Supplementary Fig. 3D). Finally, values smaller than the 0.5 th and bigger than 99.5th percentile were discarded to avoid artifactual biases. The resulting distributions are shown in Fig. 2c. Before exposure to IR, the interquartile distance (IQD) of the nano-foci DNA content was $\sim 23-65 \mathrm{~kb}$. On IR $(0.5 \mathrm{~h})-$ after $\gamma \mathrm{H} 2 \mathrm{AX}$ spreading - it increased to $\sim 34-159 \mathrm{~kb}$, with a median length of $75 \mathrm{~kb}$ (Fig. $2 \mathrm{c}$ and Supplementary Tables 1 and 2).

To provide another line of evidence supporting our 3D-SIM metrics, we produced $\gamma \mathrm{H} 2 \mathrm{AX}$ ChIP-Seq profiles under the same experimental conditions employed for the microscopic analysis. Next, we integrated the genomic data with the super-resolution microscopy data to establish a novel combined approach (described in detail in the Methods and Supplementary Fig. 4) and, thus, provide estimates of the $\gamma \mathrm{H} 2 \mathrm{AX}$-decorated chromatin domain size. Overall, the resulting $\gamma \mathrm{H} 2 \mathrm{AX}$ genomic domains' size was in good agreement with that of 3D-SIM $\gamma \mathrm{H} 2 \mathrm{AX}$ nano-foci, although the former were $\sim 30 \%$ smaller (IQD: $10-110 \mathrm{~kb}$ at $0.5 \mathrm{~h}$ ). Because our approach only takes into account the in cis contribution to the size of the genomic domains, the difference between the latter and those measured by 3D-SIM can be attributed to inter-chromosomal contribution ${ }^{23}$.

The DDR uncouples histone modifications and DNA compaction. To characterize the (epi)genetic composition of $\gamma \mathrm{H} 2 \mathrm{AX}-$ decorated chromatin during DDR, we related the ChIP-Seq $\gamma \mathrm{H} 2 \mathrm{AX}$ profiles to multiple genomic features, (Supplementary Table 3). First, we computed the density of such genomic features as well as the abundance of $\gamma \mathrm{H} 2 \mathrm{AX}$ in $10 \mathrm{~kb}$ genomic intervals. Next, we calculated the genome-wide Spearman's $\rho$ correlation coefficient of each feature with $\gamma \mathrm{H} 2 \mathrm{AX}$ profiles before and during the DDR (Fig. 3a). The outcome of the analysis showed a strong correlation at early time post IR between $\gamma \mathrm{H} 2 \mathrm{AX}$ and euchromatic features such as GC content (Supplementary Fig. 5A; maximum Spearman's $\left.\rho: 0.81, P<2.2 \times 10^{-16}\right)$, DNase hypersensitivity sites, Regions of IncreaseD Gene Expression (RIDGEs), early replication timing and histone modifications associated with transcriptionally active chromatin state (for example, H3K36me3, H3K4me1/2/3 and H3K9ac). Heterochromatic features, such as AT content (Topo.CAT-YTA-RAK motif), lamin-binding sites, late replication timing, intensity of Giemsa shades and H3K9me3, were negatively correlated to $\gamma \mathrm{H} 2 \mathrm{AX}$, instead. Notably, this trend was inverted at later times, with heterochromatic features correlating to residual $\gamma \mathrm{H} 2 \mathrm{AX}$ levels. An exemplary $\gamma \mathrm{H} 2 \mathrm{AX}$ profile on chromosome 21 is shown in Fig. 3b. Quantification of $\gamma \mathrm{H} 2 \mathrm{AX}$ levels, before and during DDR, in (anti-)RIDGEs, Giemsa shades as well as in $\mathrm{H} 3 \mathrm{~K} 36 \mathrm{me} 3-$ and $\mathrm{H} 3 \mathrm{~K} 9 \mathrm{me} 3$-decorated chromatin domains is shown in Supplementary Fig. 5B-D and Supplementary Table 4.

To validate and extend these findings at the single-cell level, we recorded 3D-SIM images of $\gamma \mathrm{H} 2 \mathrm{AX}$ immunofluorescence combined with either H3K36me3 or H3K9me3 labelling (Fig. 4a). These two histone modifications recapitulate the results from Fig. 3a, with the former being mainly associated with actively transcribed genes ${ }^{24}$, while the latter is abundant in heterochromatic (for example, pericentromeric regions) and transcriptionally silent regions ${ }^{25}$. We segmented $\gamma \mathrm{H} 2 \mathrm{AX}$ nano-foci as previously described and, in addition, we measured the $\mathrm{H} 3 \mathrm{~K} 36 \mathrm{me} 3$ or $\mathrm{H} 3 \mathrm{~K} 9 \mathrm{me} 3$ fluorescence intensity in the volume occupied by $\gamma \mathrm{H} 2 \mathrm{AX}$ nano-foci. In the latter, H3K36me3 signal was high at early time points, but not at $24 \mathrm{~h}$ post IR, as opposed to $\mathrm{H} 3 \mathrm{~K} 9 \mathrm{me} 3$ signal, which was low at early time points but higher $24 \mathrm{~h}$ post IR (Fig. $4 \mathrm{~b}$ ). We observed similar results when measuring $\gamma \mathrm{H} 2 \mathrm{AX}$ fluorescence intensity in the volume of H3K36me3- and H3K9me3-decorated chromatin (Fig. 4c). Together, these findings recapitulate our genomic results, indicating that $\gamma \mathrm{H} 2 \mathrm{AX}$ nano-foci are mainly associated to an active chromatin state during the early and mid-stages of DDR, whereas the residual phosphorylation signal is enriched in heterochromatin at later times.

Based on these data, we expected an enrichment of $\gamma \mathrm{H} 2 \mathrm{AX}$ nano-foci in compact chromatin (that is, DAPI-dense structures) at later times. However, the mean DAPI content of $\gamma \mathrm{H} 2 \mathrm{AX}$ nano-foci remained unvaried over the time, and, if at all, was lower at $24 \mathrm{~h}$ (Fig. $4 \mathrm{~d}$ ). In fact, $\gamma \mathrm{H} 2 \mathrm{AX}$ nano-foci were located in close proximity to DAPI-dense structures, and the two seldom overlapped. To quantify this, we measured the maximum DAPI intensity in a 3D-region dilated by three voxels in all dimensions around each $\gamma \mathrm{H} 2 \mathrm{AX}$ nano-focus, which we referred to as 'shell' (Supplementary Fig. 5E). Shells always presented higher DAPI signal than the nano-foci (Fig. 4e). This is in agreement with previous observations, whereby $\gamma \mathrm{H} 2 \mathrm{AX}$-decorated chromatin was excluded from DAPI-dense structures following DSB induction ${ }^{26,27}$. These findings prompted us to investigate the condensation state of $\mathrm{H} 3 \mathrm{~K} 9 \mathrm{me3}$-decorated chromatin after DNA damage induction. On IR, we observed a progressive decrease of DAPI intensity in $\mathrm{H} 3 \mathrm{~K} 9 \mathrm{me} 3$-decorated chromatin, up to $24 \mathrm{~h}$ (Fig. 4f). Such decrease was not observed in H3K36me3decorated chromatin. Together, this implies that heterochromatic regions underwent DNA decondensation, although they retained their histone marks. To independently validate this finding, we investigated $\gamma \mathrm{H} 2 \mathrm{AX}$ and $\mathrm{H} 3 \mathrm{~K} 9 \mathrm{me} 3$ levels before and after the induction of CRISPR-Cas9-mediated DNA DSBs targeted at heterochromatic murine major satellite repetitive DNA elements, in $\mathrm{C} 2 \mathrm{C} 12$ cells (Fig. 5a). These genomic regions are predominantly found at $\mathrm{H} 3 \mathrm{~K} 9$ me3-rich chromatin and are the most condensed chromatin domains in the mouse genome (chromocentres). As early as the ectopically expressed Cas9 was active $(>3 \mathrm{~h}), \gamma \mathrm{H} 2 \mathrm{AX}$ was visible at $\mathrm{H} 3 \mathrm{~K} 9 \mathrm{me}$-decorated chromatin (chromocentres) (Fig. 5b). Quantification of the H3K9me3 and $\gamma \mathrm{H} 2 \mathrm{AX}$ fluorescence intensity in the segmented chromocentres revealed that both signals co-localized (Fig. 5c). Next, we analysed the condensation state of Cas9-targeted chromocentres by means of dual-colour STED microscopy and DNA density measurements. On Cas9-mediated DSBs induction, chromocentres were dramatically decondensed (Fig. 5d,e). Remarkably, they retained the $\gamma \mathrm{H} 2 \mathrm{AX}$ mark, which was more abundant where the DNA signal was diminished (Fig. 5d). This observation is in agreement with our 3D-SIM data, whereby the $\gamma \mathrm{H} 2 \mathrm{AX}$ nano-foci present a partially decondensed state, with diminished DNA levels relative to their surroundings (Fig. 4f).

Taken together, these findings show that $\gamma \mathrm{H} 2 \mathrm{AX}$ nano-foci are chromatin units over represented in transcriptionally active 
a

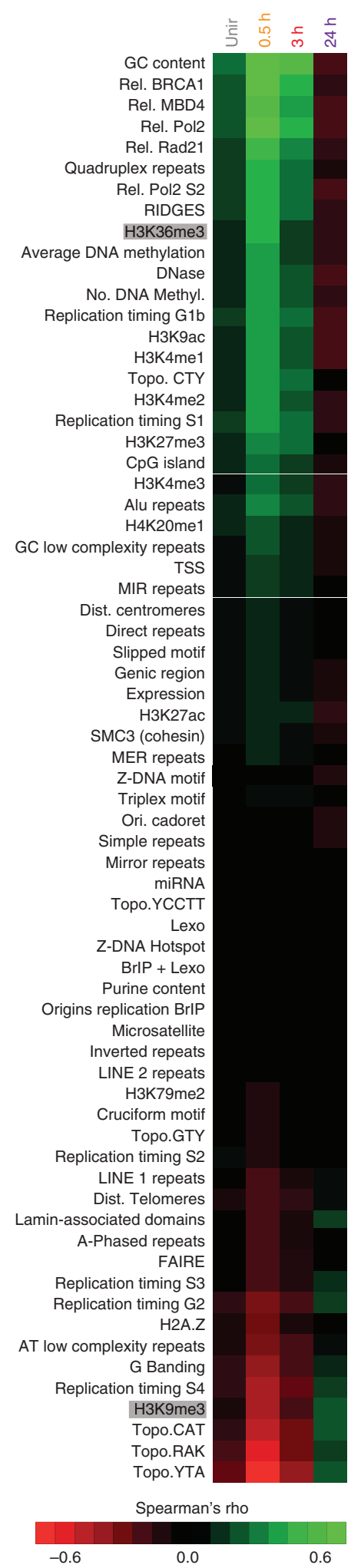

b

$\mathrm{H} 3-$

K9me3 K36me3 $\quad$ H2AX

$\begin{array}{llllll}2 & 0 & 2 & -1.0 & 0.0 & 1.0\end{array}$

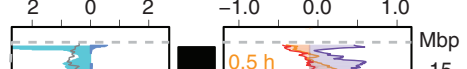

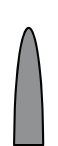

है

$24 \mathrm{~h}$ 表

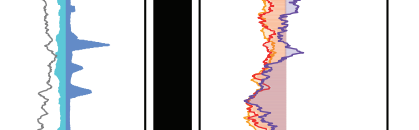

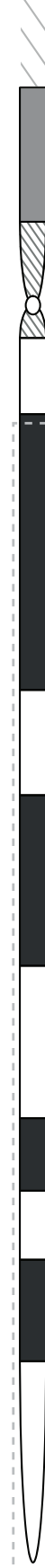
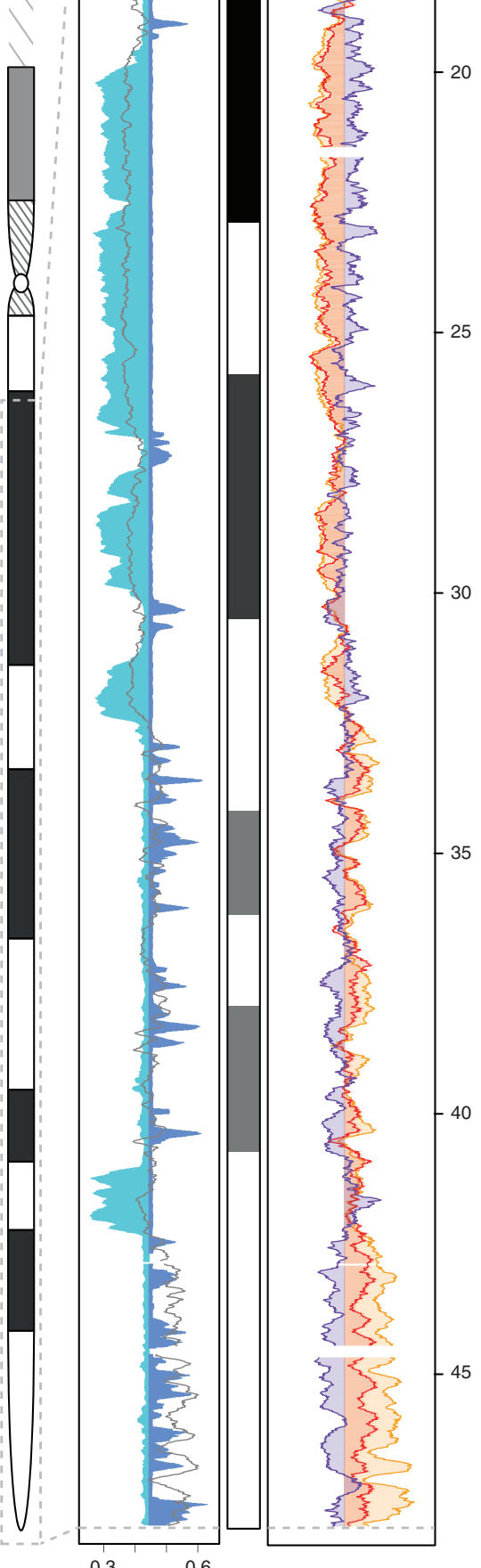

0.3

Figure 3 | Temporal correlation of $\gamma$ H2AX ChIP-Seq signal and genomic features. (a) Genome-wide correlation between ChIP-Seq $\gamma H 2 A X$ profiles and genomic features, before and during DDR. Spearman's $\rho$ correlation coefficient is calculated between $10 \mathrm{~kb}$-binned $\gamma \mathrm{H} 2 \mathrm{AX}$ profiles and the genomic features (Supplementary Table 3), and colour-coded from red (anti-correlation) to green (correlation). All genomic features are ordered decreasingly, according to the highest correlation value $\left(\gamma \mathrm{H} 2 \mathrm{AX}\right.$ and GC, $0.5 \mathrm{~h}$ : 0.81). For all correlations: $P \ll 2.2 \times 10^{-16}$. (b) Exemplary ChIP-Seq $\gamma \mathrm{H} 2 \mathrm{AX}$ profile on chromosome 21. (left) H3K9me3, H3K36me3 and GC content (grey line); (right) $\gamma \mathrm{H} 2 \mathrm{AX}$ levels during DDR. 
a
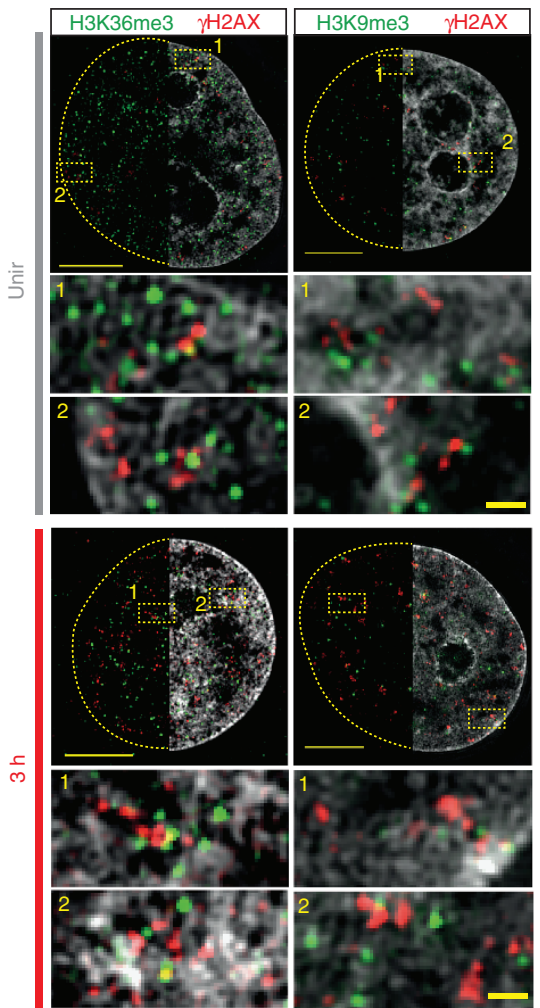

b
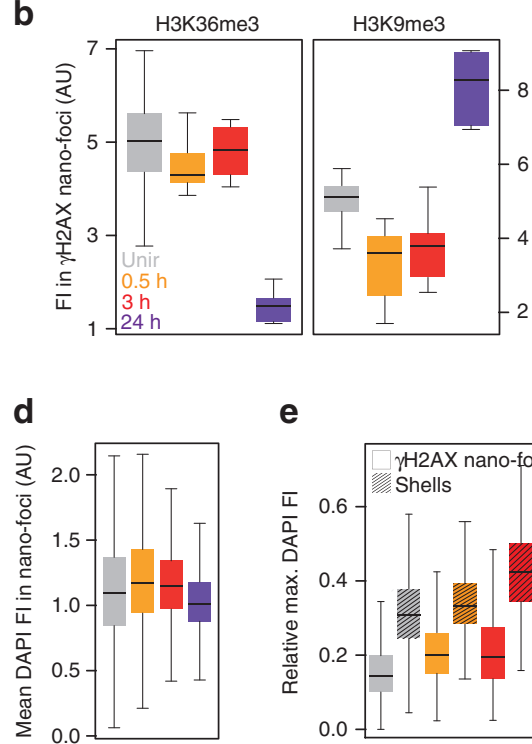

e

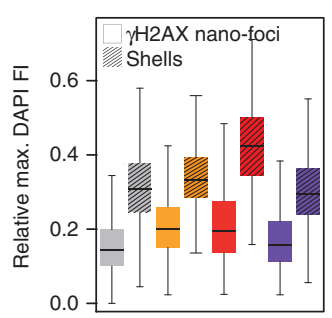

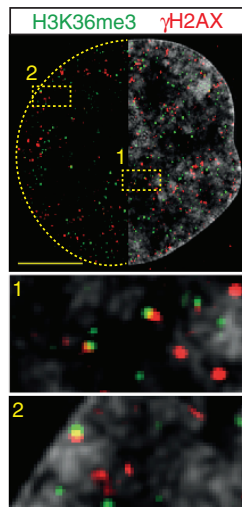
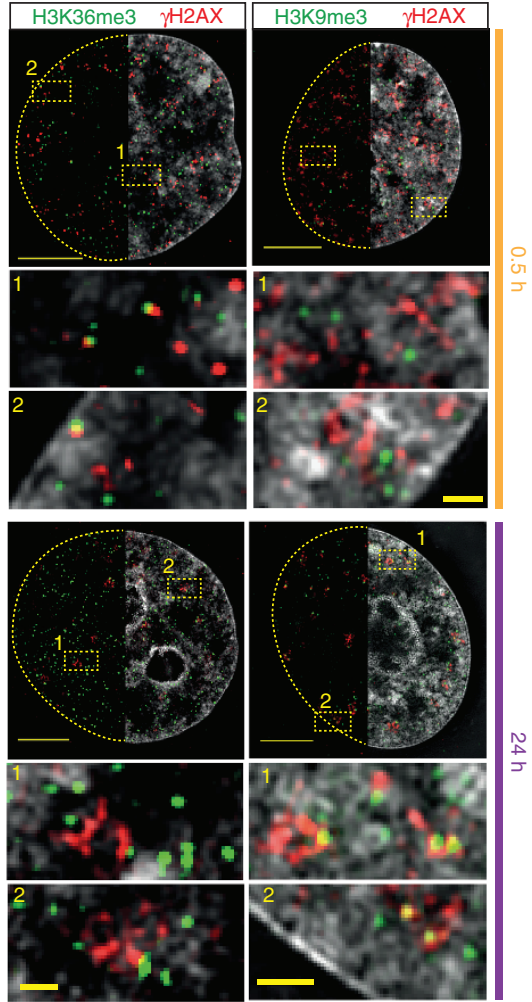

C

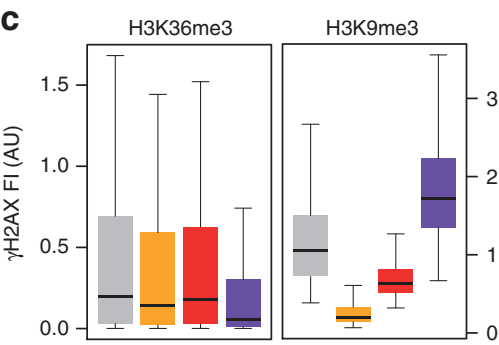

\section{f}

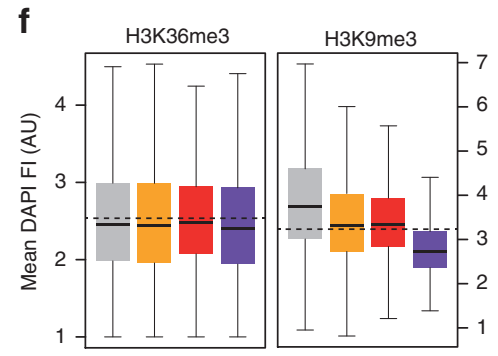

Figure 4 | 3D-SIM chromatin composition analysis of $\gamma \mathbf{H} 2 \mathbf{A X}$ nano-foci before and during DDR. (a) Exemplary 3D-SIM images of $\gamma \mathrm{H} 2 \mathrm{AX}$ (red) and H3K9me3/H3K36me3 (green) co-immunostaining before and after IR. Top panels: mid-nuclear sections showing $\gamma \mathrm{H} 2 \mathrm{AX}$ and histone marks with (right half) or without (left half) DAPI counterstaining. The dashed lines depict the nuclear contour. Bottom panels: magnification of the yellow dashed boxes with corresponding reference number. Scale bars, $5 \mu \mathrm{m}$ and $500 \mathrm{~nm}$ for main micrographs and magnified regions, respectively. (b) Quantification of the H3K36me3 and H3K9me3 fluorescence intensities measured in $\gamma \mathrm{H} 2 \mathrm{AX}$ nano-foci volumes. Kruskal-Wallis $\chi^{2}=19.875, \mathrm{df}=3, P=1.802 \times 10^{-4}$ and Kruskal-Wallis $\chi^{2}=24,451, \mathrm{df}=3, P=2.011 \times 10^{-5}$. (c) Quantification of the $\gamma \mathrm{H} 2 \mathrm{AX}$ fluorescence intensity in H3K36me3-(Kruskal-Wallis $\chi^{2}=261,960$, $\mathrm{df}=191,020, P<2.2 \times 10^{-16}$ ) and H3K9me3-(Kruskal-Wallis $\chi^{2}=246,300, \mathrm{df}=232,750, P<2.2 \times 10^{-16}$ ) decorated chromatin. (d) Mean DAPI intensity in $\gamma \mathrm{H} 2 \mathrm{AX}$ nano-foci. Kruskal-Wallis $\chi^{2}=247,910, \mathrm{df}=245,320, P=1.129 \times 10^{-4}$. (e) Quantification of maximum DAPI intensity in the volume occupied by $\gamma \mathrm{H} 2 \mathrm{AX}$ nano-foci (regular boxes) and shells (pattern), relative to the maximum integrated nuclear intensity. Shells represent 3D hollow structures surrounding $\gamma \mathrm{H} 2 \mathrm{AX}$ nano-foci (Supplementary Fig. 5E and 'Methods' section). Wilcoxon rank sum all $<2.2 \times 10^{-16}$. (f) Mean DAPI fluorescence intensity in H3K36me3- or H3K9me3-decorated chromatin. Kruskal-Wallis $\chi^{2}=303,050, \mathrm{df}=292,700, P<2.2 \times 10^{-16}$ and Kruskal-Wallis $\chi^{2}=25,500, \mathrm{df}=25,002, P=0.01338$. Dotted lines: mean DAPI intensity measured over the whole analysed nuclei. All boxes and whiskers are as in Fig. 1. AU: arbitrary units. Results are from two independent experiments. 
a

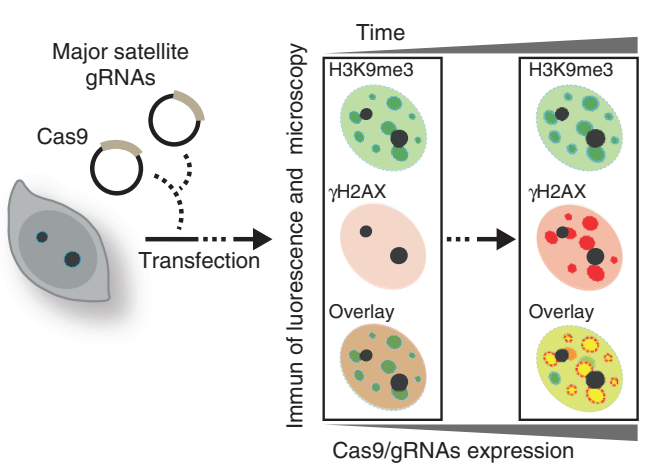

b
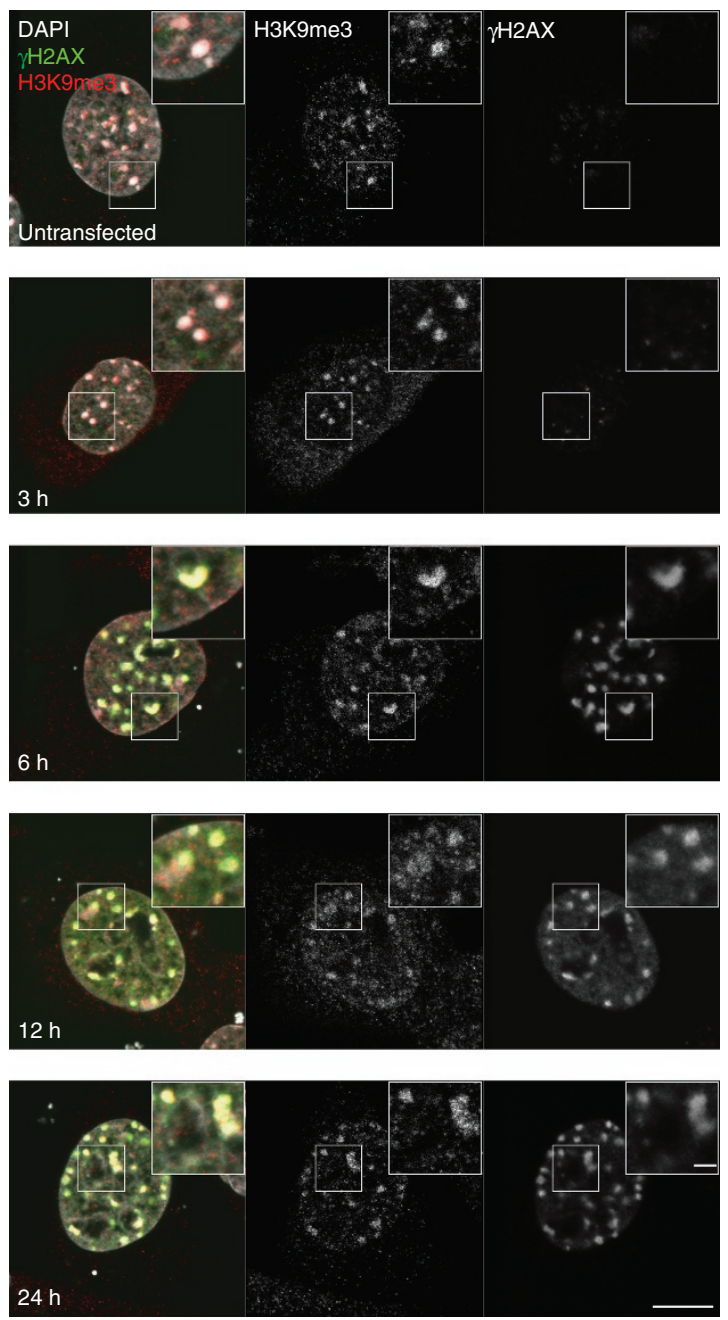

C

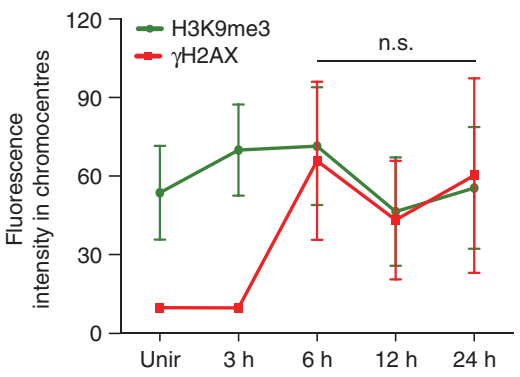

d
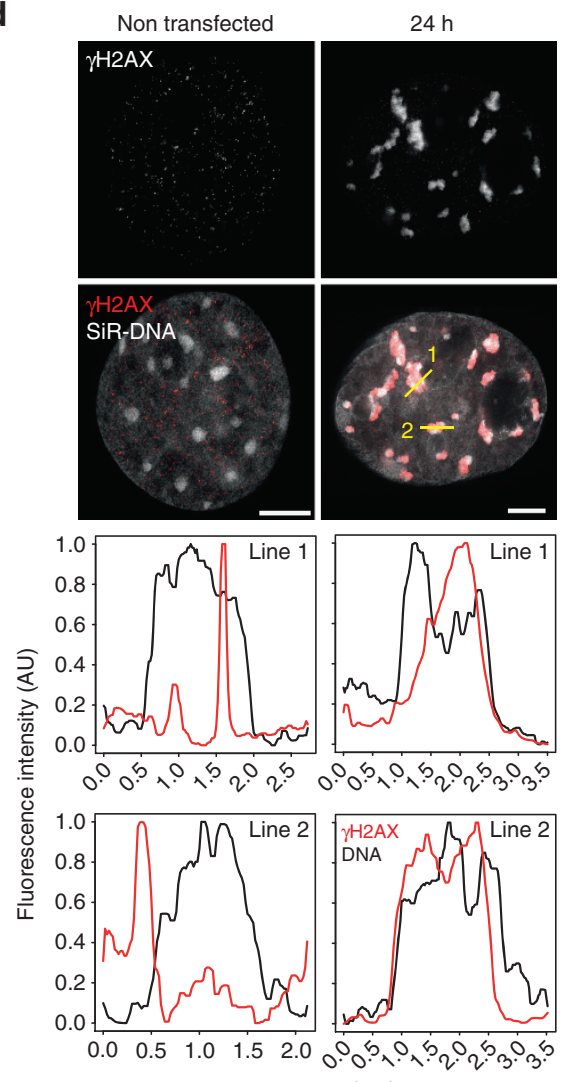

Distance $(\mu \mathrm{m})$

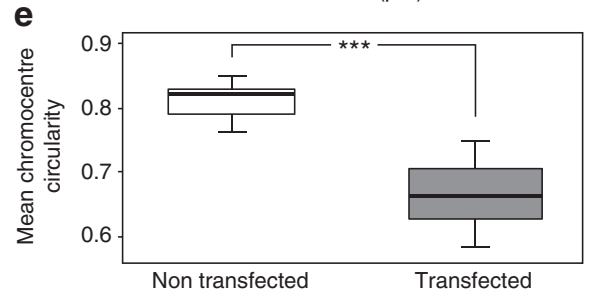

Figure 5 | Analysis of $\gamma \mathbf{H} 2 \mathrm{AX}$ and H3K9me3 levels at heterochromatin-targeted CRISPR-Cas9-mediated DSBs. (a) Schematics of the CRISPR-Cas9mediated DSBs induction at murine major satellites DNA. C2C12 cells were transfected with Cas 9 and major satellites gRNAs plasmids and fixed after the indicated times. (b) Representative immunofluorescence images of $\gamma \mathrm{H} 2 \mathrm{AX}$ and $\mathrm{H} 3 \mathrm{~K} 9 \mathrm{me} 3$ in $\mathrm{C} 2 \mathrm{C} 12$ cells. Scale bars, $10 \mu \mathrm{m}$ and $2 \mu \mathrm{m}$ for micrograph and inset, respectively. (c) Quantification of $\gamma \mathrm{H} 2 \mathrm{AX}$ and $\mathrm{H} 3 \mathrm{~K} 9 \mathrm{me} 3$ fluorescence intensity from DAPI-segmented chromocentres. Mean and s.d. from (b) are shown. $n=5$ cells ( $2-19$ chromocentres), 5 cells (6-15 chromocentres), 5 cells (8-19 chromocentres), 5 cells (13-19 chromocentres) and 4 cells (9-19 chromocentres), for untransfected, $3 \mathrm{~h}, 6 \mathrm{~h}, 12 \mathrm{~h}$ and $24 \mathrm{~h}$ time points, respectively. See image analysis in the 'Methods' section for details.

(d) Representative STED immunofluorescence images of $\gamma \mathrm{H} 2 \mathrm{AX}$ and SiR-labelled DNA as indicated. Yellow lines: line profiles (shown below). For the latter, fluorescence intensities were normalized to the min-max range of values of each profile. Lines were smoothed by a 5-window running median.

(e) Chromocentres decondensation after major satellite-targeted Cas9, assessed as mean chromocentre circularity in transfected $(n=9)$ and untransfected $(n=10)$ cells. For each cell, the circularity of chromocentres $\left(>100 \mathrm{px}^{2}\right)$ within the nucleus was determined as described in the 'Methods' section, yielding shape information for 165 (transfected cells) and 148 (untransfected cells) chromocentres. Statistics: Wilcoxon rank sum test $\left({ }^{\star \star \star} P<10^{-3}\right)$. Scale bar, $2 \mu \mathrm{m}$. 
regions early on exposure to IR. During the late stage of DDR, they mark heterochromatic regions whose DNA is in a locally decondensed state while keeping the characterizing histone marks (for example, H3K9me3). We propose that by retaining their histone mark, the chromatin identity of such domains is preserved. This not only indicates that the actual chromatin compaction state can be uncoupled from the histone modifications of a given chromatin domain, but also it suggests a modality to reestablish the original chromatin state, once DNA repair is accomplished.

$\gamma \mathrm{H} 2 \mathrm{AX}$ foci consist of spatially clustered $\gamma \mathrm{H} 2 \mathrm{AX}$ nano-foci. On exposure to IR, and as DDR progressed, $\gamma \mathrm{H} 2 \mathrm{AX}$ nano-foci were distributed throughout the nuclear volume, though they appeared to be spatially clustered (Fig. 6a and Supplementary Fig. 6A). To investigate such spatial clustering, we reconstructed the position of $\gamma \mathrm{H} 2 \mathrm{AX}$ nano-foci in the 3D nuclear space by collecting their 3D coordinates. Next, we measured the distances between the centroid of each nano-focus and all the other nano-foci in the nucleus. If the centroids of two objects were closer than a given cutoff distance, we assigned the corresponding nano-foci to the same cluster (Fig. 6b and Supplementary Fig. $1 \mathrm{H}$ and 'Methods' section). Based on the median lateral nano-focus radius of $\sim 100 \mathrm{~nm}$, we reasoned that two adjacent nano-foci would be spatially positioned so that their centroids would be at least $200 \mathrm{~nm}(2 \times$ radius $)$ away. Indeed, cutoff distances smaller than $300 \mathrm{~nm}$ resulted in poor clustering (Supplementary Fig. 6B). Similarly, distances bigger than $700 \mathrm{~nm}$ reduced the number of clusters at all time points, cancelling out differences over the time and, hence, impeding the analysis of the repair kinetics (Supplementary Fig. 6B). A cutoff distance of $500 \mathrm{~nm}$ (Fig. 6b) resulted in the highest number of clusters and a clear repair kinetics (Fig. 6c and Supplementary Table 5). Overall, the number of clusters was significantly higher than that of foci resolved by confocal microscopy, and comparable to the number of foci observed in pseudo-wide-field images (Fig. 1b,d). After IR, clusters were composed of a median number of four nano-foci (Fig. 6d), with the distributions remaining remarkably similar for all time points. This indicates that at times when the DSBs are repaired, the complete clusters, rather than single nano-foci, are removed en bloc. Coherently, clusters had an integrated median volume of about $0.05 \mu \mathrm{m}^{3}$ (Supplementary Fig. 6C), which decreased at later times. The average inter-centroid distance measured between all nano-foci belonging to a given cluster, the shortest path connecting all the centroids in a given cluster, and the inter-focal volume delimited by the 3D coordinates of the centroids of each nano-focus belonging to a cluster showed similar kinetics (Fig. 6b and Supplementary Fig. 6D-F). In all cases, these parameters increased after IR and then decreased, indicating that the nano-foci in each cluster were progressively closer to one another as the DDR progressed. One possible explanation is an active chromatin structure change bringing the clustered nano-foci in close proximity and, thus, facilitating the repair process of complex lesions at later times. However, the possibility that the clusters repaired at later times might correspond to a subset of damaged chromatin fibres whose location was in close spatial proximity already at earlier times is equally possible.

Finally, based on the previous nano-foci DNA content estimates, we calculated the DNA content of clusters by summing the DNA content of all $\gamma \mathrm{H} 2 \mathrm{AX}$ nano-foci belonging to a given cluster (Supplementary Tables 1 and 2). After IR, we observed broad-size distributions, with IQDs of about 197-938, 137-622 and $112-554 \mathrm{~kb}$ for $0.5 \mathrm{~h}, 3 \mathrm{~h}$ and $24 \mathrm{~h}$ time points, respectively (Fig. 6e). Overall, the cluster DNA content is in the (sub-)Mbp range, being directly relevant to genome regulation processes, as reported by genomic ${ }^{14,15,17}$ or super-resolution microscopy ${ }^{28}$ methods.

In view of these findings, and taking into account that the cutoff distance we applied for the cluster analysis is comparable in size to the $\gamma \mathrm{H} 2 \mathrm{AX}$ objects segmented in the pseudo-wide-field images (Supplementary Fig. 3A), we conclude that $\gamma \mathrm{H} 2 \mathrm{AX}$ foci, as previously identified by conventional microscopy techniques, correspond to spatially organized clusters, composed of several distinct nano-foci of phosphorylated H2AX in close spatial proximity whose pattern in the nucleus depends on the progression of DDR. While clusters are chromatin higher-order organization units in the half-a-megabase-pair size range, nano-foci are lower-order chromatin organization units whose size spans $40-160 \mathrm{~kb}$.

$\gamma \mathrm{H} 2 \mathrm{AX}$ clusters contain single DNA DSBs. As previously reported, in higher eukaryotes ${ }^{6-8}, \gamma \mathrm{H} 2 \mathrm{AX}$ is proposed to spread up to Mbps from the lesion site in a non-homogenous non-symmetrical fashion ${ }^{11,12}$. This implies that $\gamma \mathrm{H} 2 \mathrm{AX}$ may also be found reasonably far from the actual DNA break. Indeed, on severe localized DNA damage (for example, caused by accelerated charged particles), pan-nuclear $\mathrm{H} 2 \mathrm{AX}$ phosphorylation is promptly induced by ATM and DNA-PK ${ }^{29}$. It is then obvious that not all $\gamma \mathrm{H} 2 \mathrm{AX}$-decorated chromatin contains a DNA DSB in the immediate vicinity.

Based on the linear increase of $\gamma \mathrm{H} 2 \mathrm{AX}$ nano-foci numbers, we observed up to 10 Gy (Supplementary Fig. 6G), and on the assumption that 1 Gy X-ray induce $30-55$ DSBs per diploid human genome $\mathrm{e}^{30-33}$, we estimated that $10 \mathrm{~Gy} \mathrm{X}$-ray would result in 470-860 DSBs in the ploidy-adjusted genome. Such numbers are conspicuously close to the number of $\gamma \mathrm{H} 2 \mathrm{AX}$ clusters we observed on IR (95\% confidence interval of median cluster number at $0.5 \mathrm{~h}$ : 767-1,133; Fig. $6 \mathrm{c}$ and Supplementary Fig. $6 \mathrm{H})$

To directly estimate the number of DNA DSBs before and during the DDR, we recorded 3D-SIM super-resolution images of immunofluorescently labelled phospho-Ku70 proteins, which are directly associated to the broken ends, together with $\gamma \mathrm{H} 2 \mathrm{AX}$. As shown in Fig. 7a,b, most of the phospho-Ku70 signal was surrounded by several $\gamma \mathrm{H} 2 \mathrm{AX}$ nano-foci. Remarkably, the number of phospho-Ku70 focal structures matched with good agreement that of our previously measured clusters (Fig. 7c). Also, the slopes of the linear regression lines computed while fitting the number of phospho-Ku70 and $\gamma \mathrm{H} 2 \mathrm{AX}$ nano-foci or clusters indicate that we measured $\sim 3.4 \quad \gamma \mathrm{H} 2 \mathrm{AX}$ nano-foci per phospho-Ku70 focal structure, or in other words, that there are $\sim 1.3$ phospho-Ku70 focal structures per $\gamma \mathrm{H} 2 \mathrm{AX}$ cluster (Fig. 7d,e). We observed similar results by assessing the number of DNA DSBs by terminal deoxynucleotidyl transferase dUTP nick end-labelling (TUNEL). TUNEL signal was often surrounded by several $\gamma \mathrm{H} 2 \mathrm{AX}$ nano-foci (Fig. $7 \mathrm{f}, \mathrm{g}$ ) and the number of TUNEL focal structures recapitulates the DDR (Fig. 7h). Finally, we observed a robust agreement between the numbers of TUNEL focal structures and phosphoKu70 (Fig. 7i) or $\gamma \mathrm{H} 2 \mathrm{AX}$ clusters (Fig. 7j). Together, these data demonstrate that $\gamma \mathrm{H} 2 \mathrm{AX}$ clusters are $\gamma \mathrm{H} 2 \mathrm{AX}$-decorated multi-unit chromatin structures containing a single DNA DSB.

CTCF delimits phosphorylated H2AX chromatin domains. Altogether, the structural features we described about $\gamma \mathrm{H} 2 \mathrm{AX}$ clusters underpin the role of a structural organization factor in regulating their formation and kinetics. CTCF is involved in diverse cellular processes, including V(D)J recombination ${ }^{34}$, regulation of transcription ${ }^{35,36}$ and replication ${ }^{17}$. It mainly acts as a regulator of chromatin architecture $e^{37,38}$ by forming and keeping chromatin loops, and the presence of CTCF-binding motif close to the boundaries of large looping chromatin domains 
a

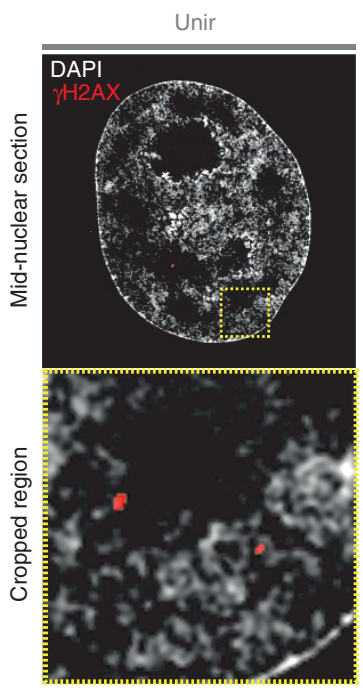

b

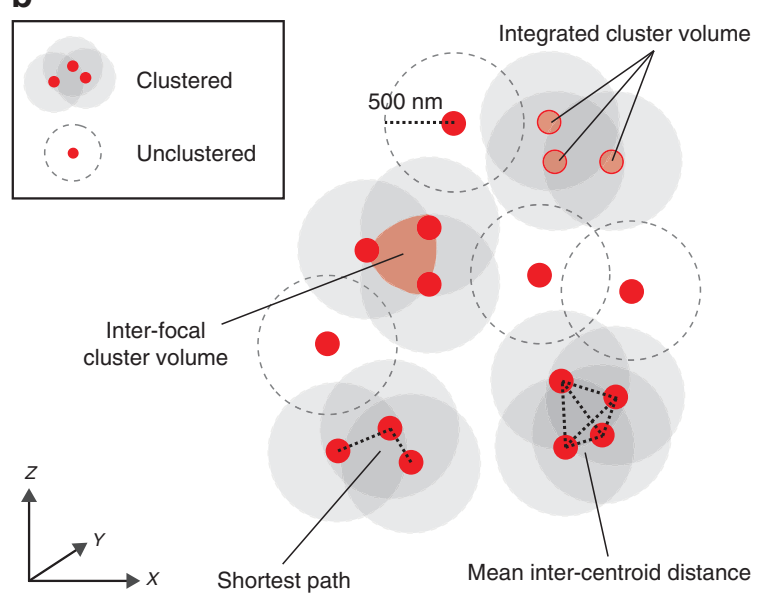

$0.5 \mathrm{~h}$

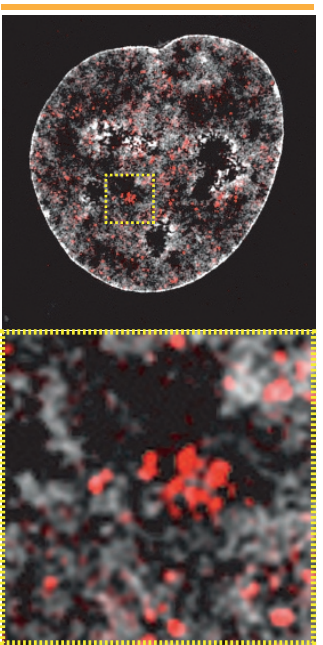

(2)
$3 \mathrm{~h}$

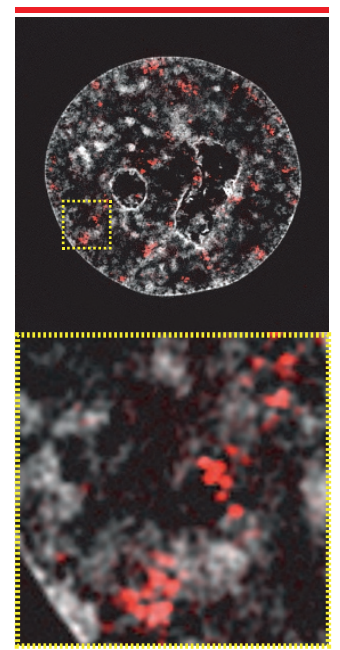

C

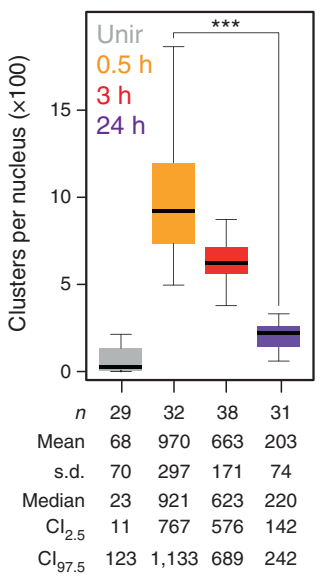

$24 \mathrm{~h}$

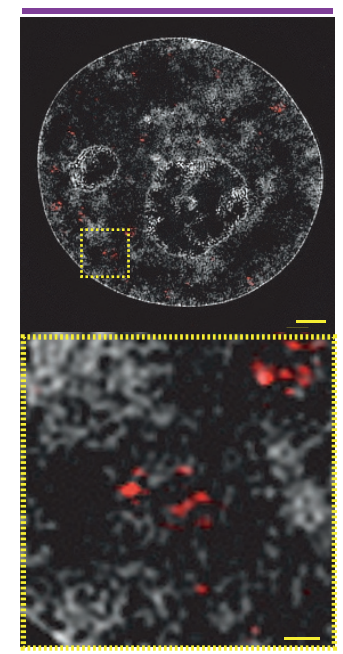

d

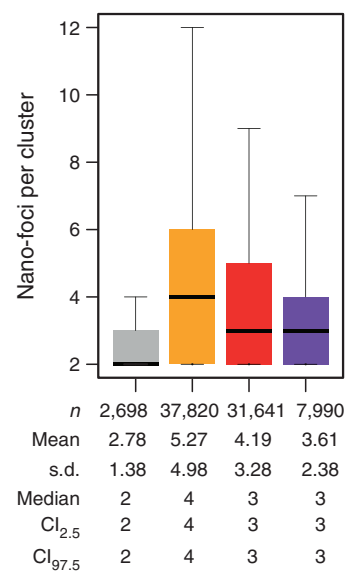

e

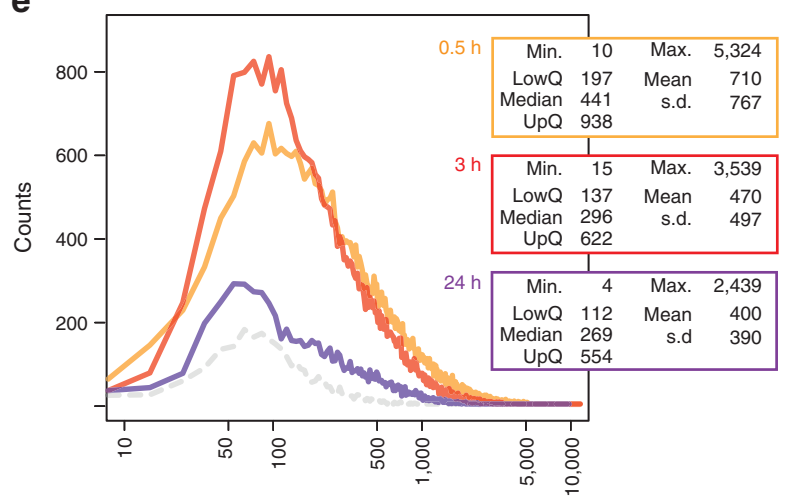

Cluster DNA content (kbp)

Figure 6 | Analysis of $\gamma \mathbf{H} 2 A X$ nano-foci spatial clustering. (a) Exemplary 3D-SIM images of $\gamma H 2 A X$ immunofluorescence before and during DDR. Shown are the mid-nuclear section with DAPI and $\gamma \mathrm{H} 2 \mathrm{AX}$ signals, and magnified view from the yellow frame. Scale bars, $2 \mu \mathrm{m}$ and $400 \mathrm{~nm}$ for main micrographs and magnified regions, respectively. (b) Schematics of $\gamma \mathrm{H} 2 \mathrm{AX} 3 \mathrm{D}$-clusters analysis. All centroids (red dots) within a sphere defined by a given cutoff radius $(500 \mathrm{~nm}$ in further analysis) are included in a cluster. For all nano-foci belonging to each given cluster, the sum of the volume of single nano-foci (integrated cluster volume), the volume delimited by the centroids (inter-focal volume), the shortest path connecting all centroids as well as the mean distance between centroids (mean inter-centroid distance) are computed (Supplementary Fig. 6C-F). (c) $\gamma \mathrm{H} 2 \mathrm{AX}$ 3D-clusters per nucleus. One-way ANOVA with Dunnett's correction; ${ }^{\star \star \star} P<10^{-3}$. (d) $\gamma \mathrm{H} 2 \mathrm{AX}$ nano-foci per 3D-clusters. Kruskal-Wallis $\chi^{2}=1,926.3, \mathrm{df}=3, P<2.2 \times 10^{-16}$. (e) DNA content distributions of $\gamma \mathrm{H} 2 \mathrm{AX} 3 \mathrm{D}$-clusters during DDR. The DNA content of each nano-focus belonging to a given cluster is summed. The dashed line depicts the distribution of $\gamma \mathrm{H} 2 \mathrm{AX} 3 \mathrm{D}$-clusters before IR. Kruskal-Wallis $\chi^{2}=5,964.1, \mathrm{df}=3, P<2.2 \times 10^{-16}$. Statistics are presented as in Fig. 2 . All boxes and whiskers are as in Fig. 1. n: number of analysed cells (c) or 3D-clusters (d). 
a

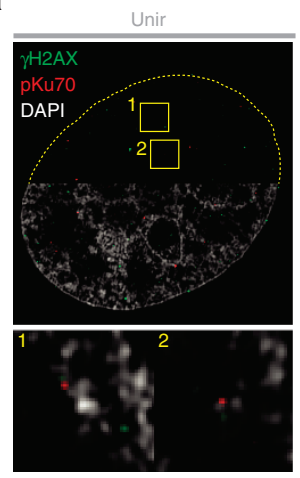

b

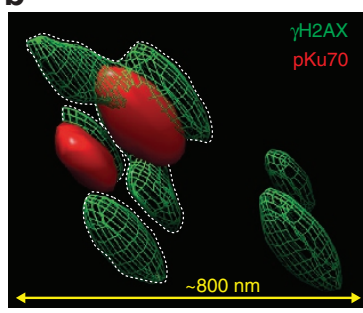

f

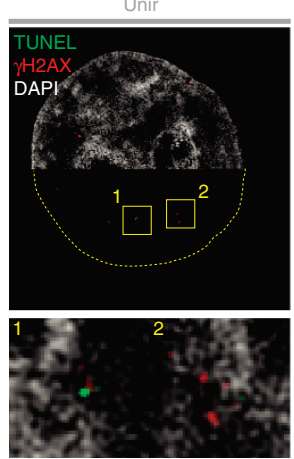

g

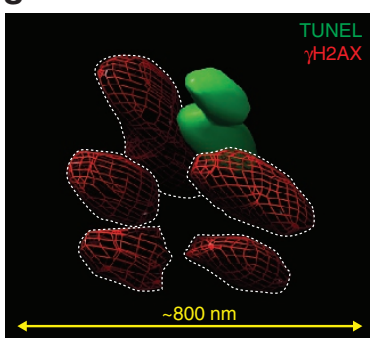

$0.5 \mathrm{~h}$

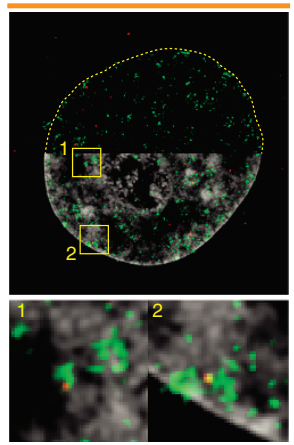

c

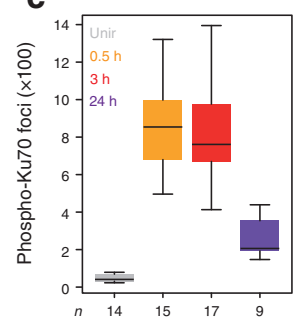

$\begin{array}{llll} & 14 & 15 & 17\end{array}$

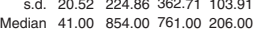

$\begin{array}{lll}\mathrm{Cl}_{2.5} & 29.00 \quad 654.00670 .00 \quad 184.00\end{array}$ $\mathrm{Cl}_{97.5} 74.001,042.00976 .00 \quad 386.00$
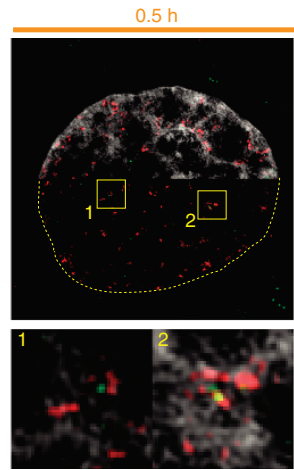

h

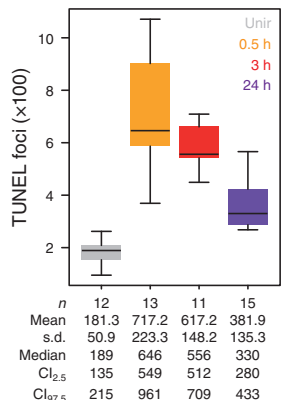

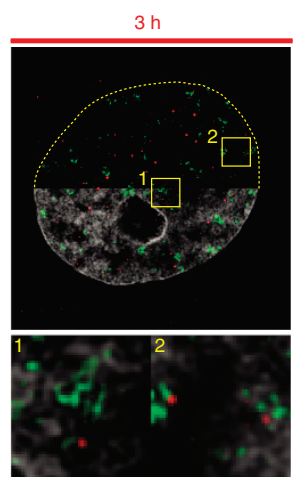
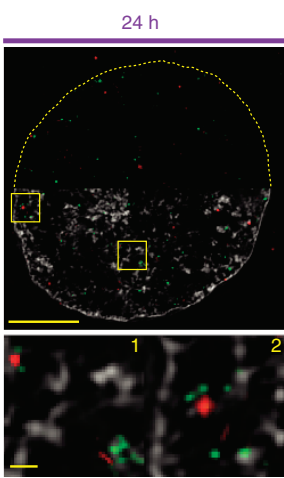

d

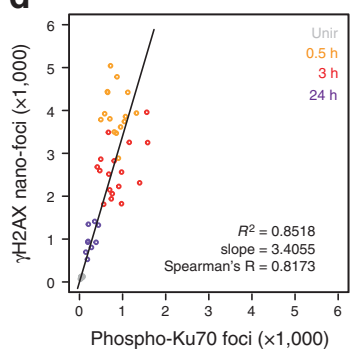

e
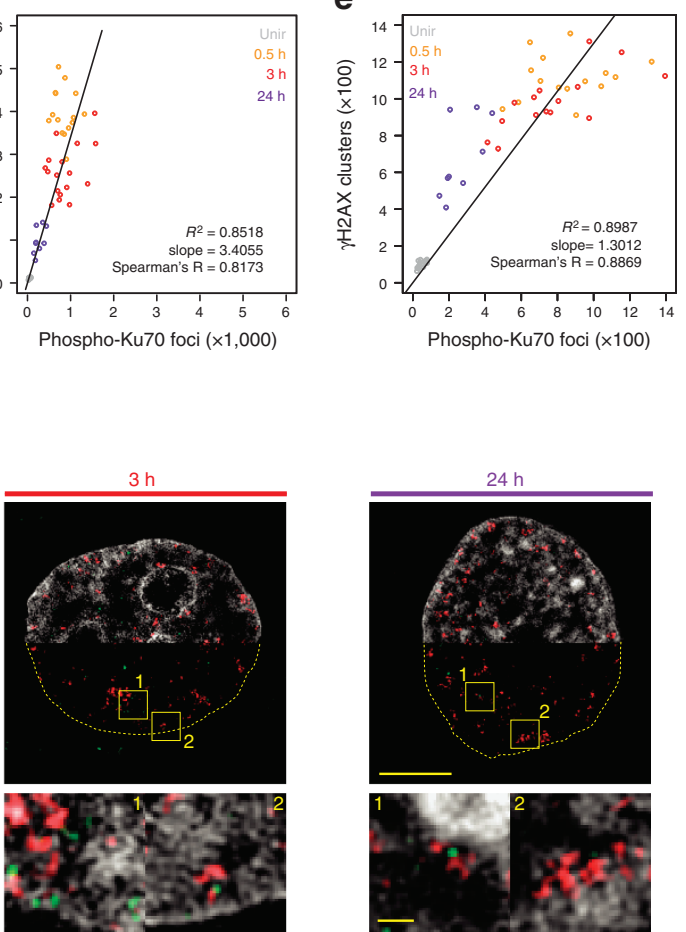

i
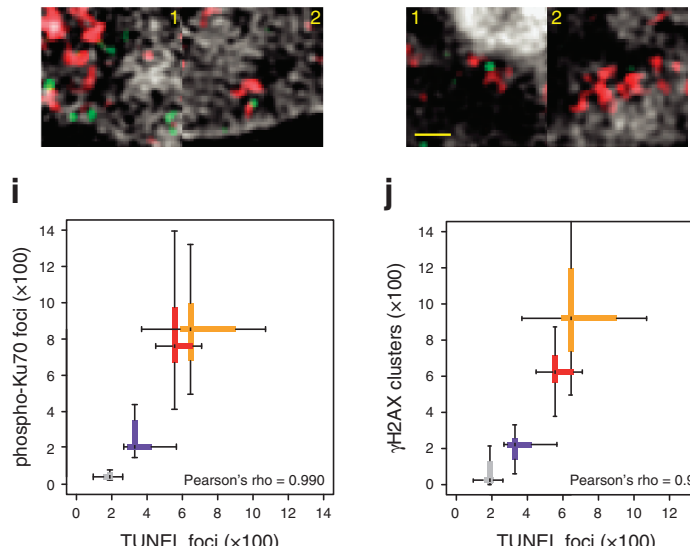

j

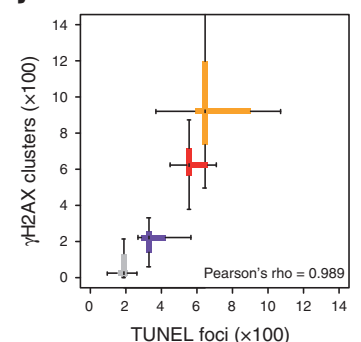

Figure 7 | Single phospho-Ku70- or TUNEL-labelled DNA DSBs are embedded in $\gamma \mathbf{H} 2 \mathrm{AX}$ clusters. (a) Exemplary 3D-SIM images of $\gamma H 2 \mathrm{AX}$ and phospho-ku70 immunofluorescence before and during DDR. Shown are the mid-nuclear section (top) and enlarged views from the yellow frames (bottom). (b) 3D rendering of $\gamma \mathrm{H} 2 \mathrm{AX}$ and phospho-Ku70 immunostaining, $24 \mathrm{~h}$ after IR. (c) Phospho-Ku70 foci number distributions before and during DDR, from 3D-SIM images (one-way ANOVA with Dunnett's correction: $\mathrm{P}<10^{-3}$ ). Scatter plots of phospho-Ku70 foci and $\gamma \mathrm{H} 2 \mathrm{AX}$ nano-foci (d) or $\gamma \mathrm{H} 2 \mathrm{AX}$ clusters (e). Each dot represents a single-cell nucleus. (f) Exemplary 3D-SIM images of $\gamma \mathrm{H} 2 \mathrm{AX}$ and TUNEL immunofluorescence before and during DDR. Shown are the mid-nuclear section (top) and enlarged views from the yellow frames (bottom). (g) 3D rendering of $\gamma \mathrm{H} 2 \mathrm{AX}$ and TUNEL immunostaining, 24 $\mathrm{h}$ after IR. (h) TUNEL foci number distributions before and during DDR, from 3D-SIM images (one-way ANOVA with Dunnett's correction: $P<10^{-3}$ ). Comparison between TUNEL and phospho-Ku70 (i) or $\gamma \mathrm{H} 2 \mathrm{AX}$ clusters (j) distributions, before and during DDR $\left(P<10^{-3}\right)$. Scale bars, $5 \mu \mathrm{m}$ and $500 \mathrm{~nm}$ for main micrographs and magnified regions, respectively. All boxes and whiskers are as in Fig. 1. n: number of analysed cells. Results are from two independent experiments. 
has been recently confirmed by in situ $\mathrm{Hi}-\mathrm{C}^{15}$. In view of these observations, and based on CTCF insulating properties, we next investigated the relationship between CTCF and $\gamma \mathrm{H} 2 \mathrm{AX}$ levels during DDR.
We identified the genomic location of putative CTCF-binding sites, based on a consensus motif modified from previous studies ${ }^{15,39}$ (Supplementary Fig. 7A). The analysis resulted in 3,909 CTCF-binding sites, separated by a median intervening
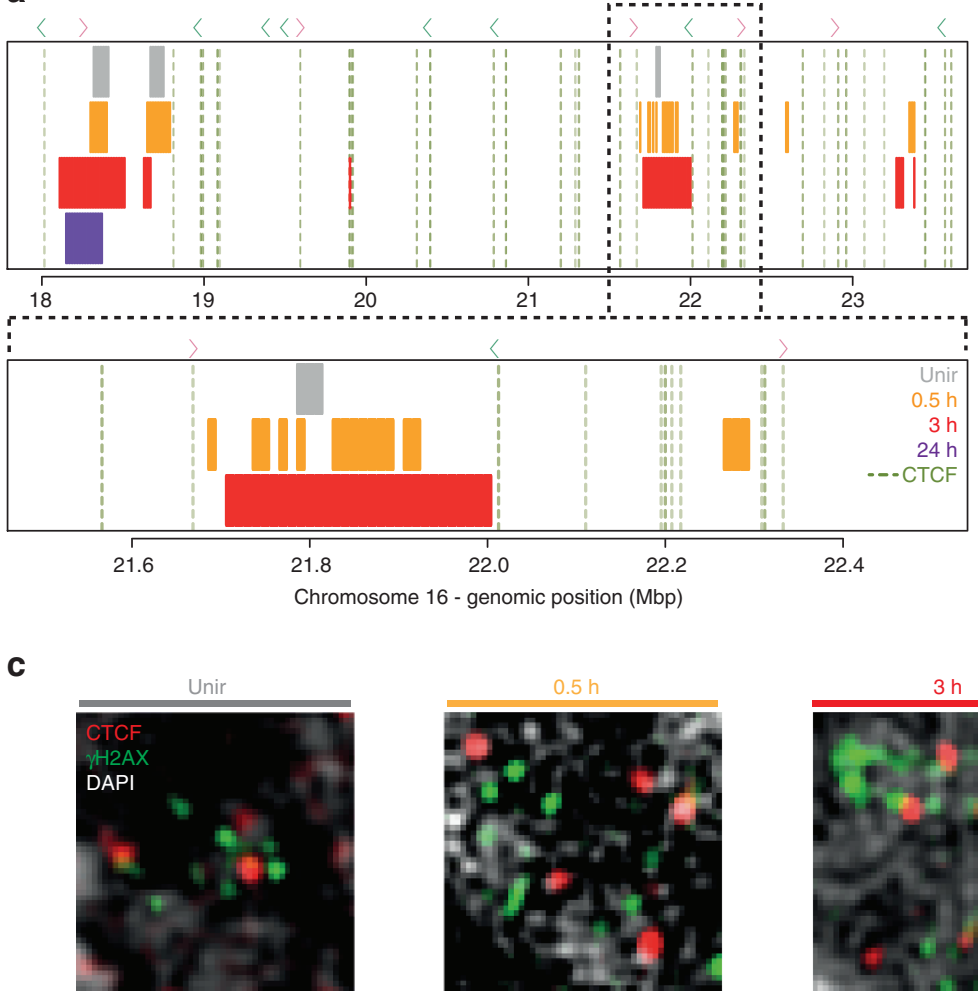

d
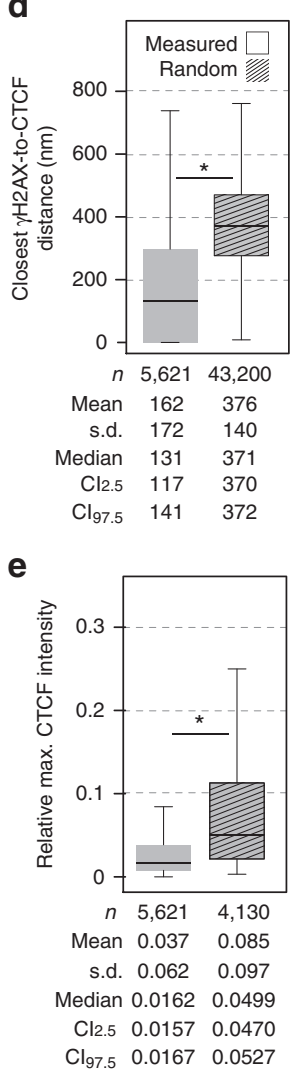
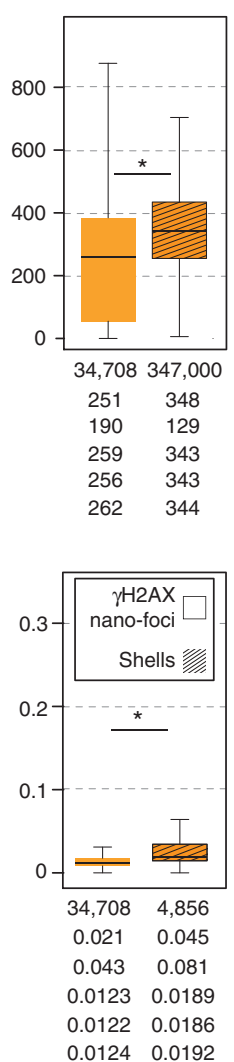

b
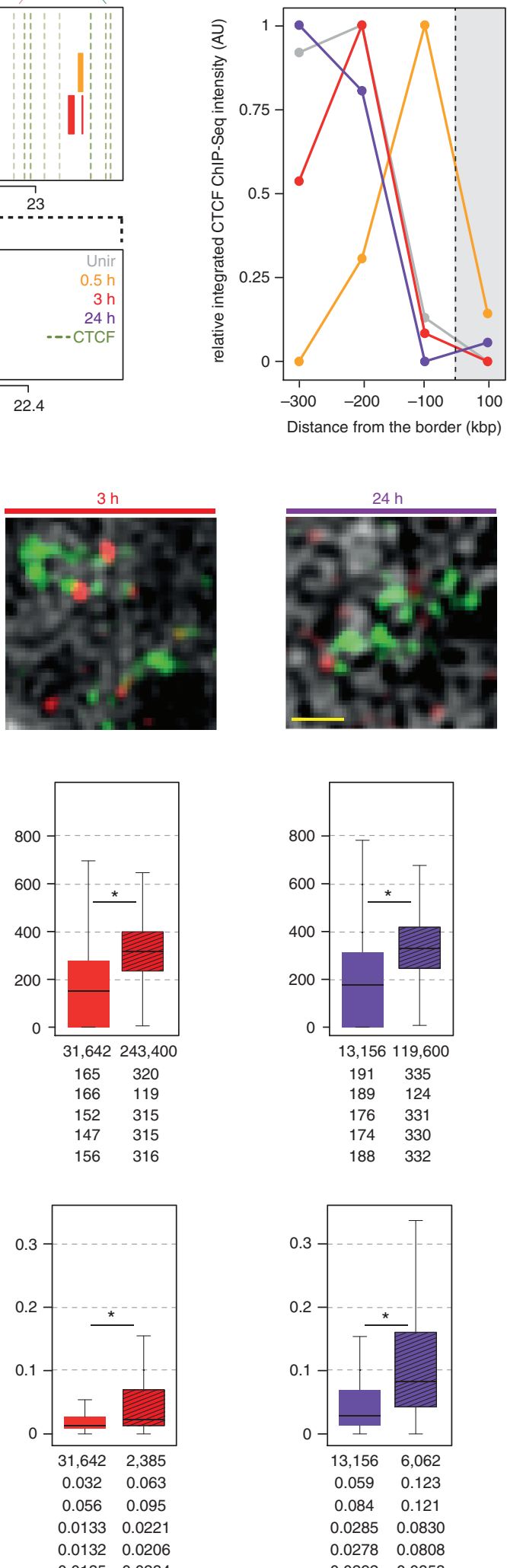
distance of $\sim 370 \mathrm{~kb}$ (IQD: 127-914 kb; Supplementary Fig. 7B). The orientation of CTCF motif had little to no impact on the measured distances (Supplementary Fig. 7B). This size range was comparable to that of $\gamma \mathrm{H} 2 \mathrm{AX}$ clusters rather than with that of single $\gamma \mathrm{H} 2 \mathrm{AX}$ nano-foci (Supplementary Fig. 7B), suggesting that individual clusters can be delimited by CTCF-binding sites. To validate such hypothesis at genomic level, we integrated our 3D-SIM-filtered $\gamma \mathrm{H} 2 \mathrm{AX}$ ChIP-Seq profiles (Supplementary Fig. 4) with publicly available HepG2 CTCF ChIP-Seq data. We identified $\sim 140,000$ CTCF genomic footprints, including CTCF occupancy levels ranging from very low to very highy. Due to the inherent nature of this ChIP-Seq data, it is unlikely that all those CTCF peaks would actually be present at the same time in a given cell. Therefore, we focused our analysis only on those CTCF genomic footprints whose occupancy score was maximum, assuming these sites would be conserved among different cell types. This reduced the number of CTCF footprints to 5,322. Remarkably, these sites were flanking most of the genomic $\gamma \mathrm{H} 2 \mathrm{AX}$ domains, before and during the DDR (Fig. 8a), yet the two signals seldom overlapped. In addition, CTCF ChIPSeq signal intensity (that is, CTCF abundance) was higher upstream or downstream of the borders of each $\gamma \mathrm{H} 2 \mathrm{AX}$ genomic domain than that computed inside the domain (Fig. 8b), indicating that high-occupancy CTCF sites function as barriers for $\gamma \mathrm{H} 2 \mathrm{AX}$ spreading.

Next, we investigated the 3D-distribution of $\gamma \mathrm{H} 2 \mathrm{AX}$ and CTCF before and during DDR at single-cell level by 3D-SIM. On IR, CTCF foci were often in the immediate proximity of $\gamma \mathrm{H} 2 \mathrm{AX}$ nano-foci (Fig. 8c and Supplementary Fig. 7C,D). The majority $(\sim 75 \%)$ of the centroid-to-centroid distances between each $\gamma \mathrm{H} 2 \mathrm{AX}$ nano-focus and the closest CTCF focal structure were within $400 \mathrm{~nm}$, and starting from $3 \mathrm{~h}$ post infrared, they all were below $200 \mathrm{~nm}$ (Fig. 8d). In all cases, the measured distances were smaller than distances between simulated random objects whose populations were comparable in numbers to those of CTCF and $\gamma \mathrm{H} 2 \mathrm{AX}$ nano-foci at each stage of DDR (Fig. 8d and Supplementary Fig. 7E). Because $\gamma \mathrm{H} 2 \mathrm{AX}$ nano-foci in our 3D-SIM images have a radius of $\sim 100 \mathrm{~nm}$, and CTCF focal structures showed comparable size, our results imply that the two objects would thus be in tight contact, with CTCF focal structures flanking $\gamma \mathrm{H} 2 \mathrm{AX}$ nano-foci. On exposure to IR, and based on the higher CTCF density in GC-rich regions, the expected $\gamma \mathrm{H} 2 \mathrm{AX}$ to-CTCF distance should be equal to, if not shorter than, that we observed in the control sample (Fig. 8d, Unir, median: $131 \mathrm{~nm}$ ). However, $0.5 \mathrm{~h}$ post IR, the median $\gamma \mathrm{H} 2 \mathrm{AX}$-to-CTCF distance was two times longer $(259 \mathrm{~nm})$. Moreover, during the late stage of the DDR, the majority of DSBs were associated to heterochromatic regions (with lower GC content). In these regions, CTCF density is lower (compared with euchromatin) and the expected $\gamma \mathrm{H} 2 \mathrm{AX}$-to-CTCF distance should be equal to, if not longer than, that we measured in a random distribution. Yet, the observed median $\gamma \mathrm{H} 2 \mathrm{AX}$-to-CTCF distance was only half of that we obtained from a random distribution (Fig. 8d, $24 \mathrm{~h}$ measured: $176 \mathrm{~nm} ; 24 \mathrm{~h}$ random: $331 \mathrm{~nm}$ ). Such close spatial proximity was confirmed by the observation that CTCF signal was more abundant in the surroundings of $\gamma \mathrm{H} 2 \mathrm{AX}$ nano-foci (as measured in the previously described shells) rather than overlapping with them (Fig. 8e and Supplementary Fig. 7F).

Taken together, our genomic and microscopy data strongly support that CTCF delimits $\gamma \mathrm{H} 2 \mathrm{AX}$ chromatin, and the two are in close spatial proximity.

CTCF is critical for spatial regulation of $\gamma \mathrm{H} 2 \mathrm{AX}$ chromatin. Finally, we investigated whether the perturbation of CTCF levels would affect the spatial distribution of $\gamma \mathrm{H} 2 \mathrm{AX}$-decorated chromatin. While CTCF knockout is lethal, a number of studies have shown neither effects on the cellular and nuclear morphology, nor in the cell cycle progression up to $72 \mathrm{~h}$ post CTCF knockdown ${ }^{40,41}$. In our experimental system, esiRNA-mediated CTCF depletion to $\sim 40 \%$ of the control protein levels (Supplementary Fig. 8A,B), resulted in a mild radiosensitization ( $20 \%$; Supplementary Fig. $8 \mathrm{C})$ and a coherent decrease (70-85\%) of CTCF foci in 3D-SIM micrographs, before and during DDR (Fig. 9a). Notably, CTCF depletion strongly impaired the formation of $\gamma \mathrm{H} 2 \mathrm{AX}$ nano-foci (Fig. 9b), which were smaller, diminished in numbers, and presented decreased volume and DNA content (Fig. 9c,d and Supplementary Fig. $8 \mathrm{D}, \mathrm{E})$. Only at $24 \mathrm{~h}$ post IR, the number of $\gamma \mathrm{H} 2 \mathrm{AX}$ nano-foci was comparable to that of the mock-knockdown samples, although with decreased fluorescence intensity, indicating a defect in the activation of the DDR. Indeed, CTCF-depleted cells showed a diminished DNA repair capability as assayed by comet single-cell analysis (Fig. 9e). Such defect was more prominent at the mid and late stages of DDR, suggesting that optimal CTCF levels are required to mount an efficient DDR. In this context, CTCF role in chromatin structural regulation may be crucial. Overall, the diminished $\gamma \mathrm{H} 2 \mathrm{AX}$ response resulted in a $\sim 2.9$-fold decrease in cluster formation (Fig. 9f). Remarkably, ATM and DNA-PKcs, the main signalling effectors involved in H2AX phosphorylation, were promptly activated on IR in both mock- and CTCF-depleted cells (Supplementary Fig. 9A,B), indicating that the presence of functional key factors of the DDR is necessary but not sufficient to trigger a proper response to DNA damage. In conclusion, we propose that CTCF, by preserving the $3 \mathrm{D}$ organization of the chromatin, is critical for the activation of an efficient DDR and, in such context, it functions as a regulator of the structural component of DDR.

\footnotetext{
Figure 8 | Genomic and microscopic analysis of CTCF spatial distribution in $\gamma \mathbf{H 2 A X}$-decorated chromatin. (a) Genomic localization of $\gamma \mathrm{H} 2 \mathrm{AX}$ ChIP-Seg domains (coloured bars) and CTCF genomic footprint (dashed green lines) in a representative region of chromosome 16. Dashed black line: magnification. Coloured arrowheads: orientation of CTCF-binding sites (red: forward; green: reverse). Details about $\gamma \mathrm{H} 2 \mathrm{AX}$ ChIP-Seq domains are in Supplementary Methods and Supplementary Fig. 4. ChIP-Seq CTCF profiles were retrieved from publicly available databases (UCSC Accession: Encode wgEH000080, wgEH000543, wgEH000401 and wgEH000470). (b) CTCF occupancy outside or inside $\gamma \mathrm{H} 2 \mathrm{AX}$ ChIP-Seq domains. The intensity of each CTCF peak in $100 \mathrm{~kb}$ bins upstream and downstream of the border of $\gamma \mathrm{H} 2 \mathrm{AX}$ ChIP-Seq domains (grey box) is summed and then presented as one-sided distribution. The bins range from \pm 300 to $\pm 200, \pm 200$ to $\pm 100, \pm 100$ to 0 and 0 to $\pm 100 \mathrm{~kb}$ (inside the domain), with 0 being the border of each domain.

AU: arbitrary unit. Genome-wide CTCF footprint localization relative to $\gamma \mathrm{H} 2 \mathrm{AX}$ ChIP-Seq domains' borders. For each domain, the distance in kb between its boundaries and the closest CTCF peak is measured and plotted as a bar (dashed lines). (c) Representative 3D-SIM images of immuno-stained $\gamma \mathrm{H} 2 \mathrm{AX}$ and CTCF before and during DDR. Scale bar, $500 \mathrm{~nm}$. (d) Quantification of the closest centroid-to-centroid distance between CTCF and $\gamma \mathrm{H} 2 \mathrm{AX}$ nano-foci from 3D-SIM images. Measured (filled boxes) and simulated (patterned boxes) distances are shown. The latter were obtained from simulated random distributions of CTCF and $\gamma \mathrm{H} 2 \mathrm{AX}$ nano-foci (100 iterations). (e) Quantification of maximum CTCF intensity in $\gamma \mathrm{H} 2 \mathrm{AX}$ nano-foci and in surrounding shells. Maximum CTCF fluorescence in the segmented space normalized over the maximum CTCF fluorescence of the entire nucleus is plotted. All boxes and whiskers are as in Fig. 1. $n$ : measured distances (d) or analysed shells (e) from two independent experiments. $\mathbf{d}, \mathbf{e}:$ Mann-Whitney test: $P<10^{-3}$.
} 


\section{Discussion}

In this study, the use of high prospecting super-resolution light microscopy technologies enabled us to identify the elementary structural units read by the DNA repair machinery, analysed as $\gamma \mathrm{H} 2 \mathrm{AX}$ focal structures following the exposure to IR.
The $\gamma \mathrm{H} 2 \mathrm{AX}$ nano-foci we identified are two- to threefold smaller-with lateral diameters of $\sim 200 \mathrm{~nm}$-and contain $\sim 10 \%$ of the conventionally estimated Mbp DNA content $^{42}$. Similar $\gamma \mathrm{H} 2 \mathrm{AX}$ substructures sizes were recently measured after heavy ion irradiation ${ }^{43}$, despite the highly
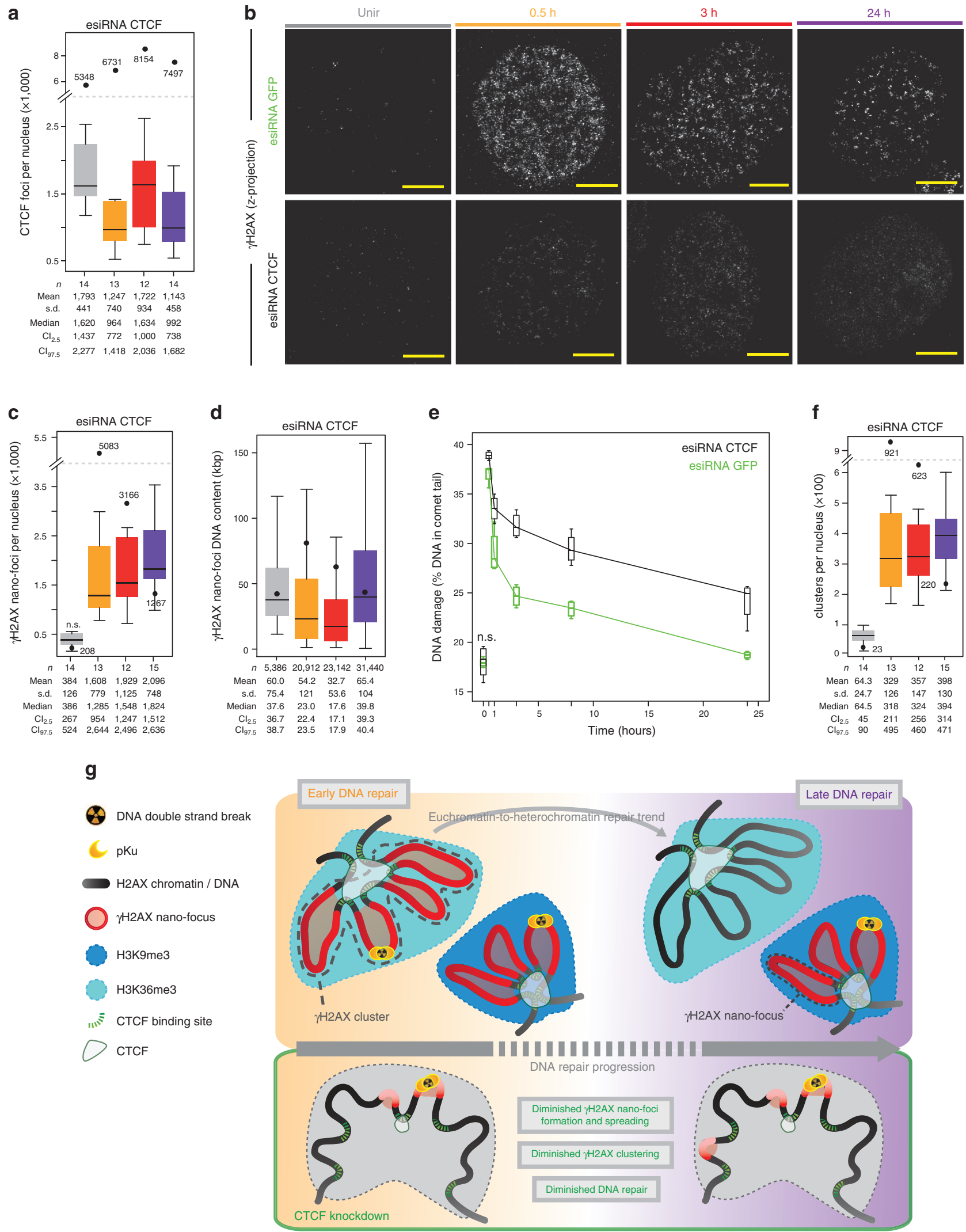
ionizing power charged particles possess, thus further supporting our findings.

Importantly, $\gamma \mathrm{H} 2 \mathrm{AX}$ nano-foci form clusters of approximately four chromatin units, and each cluster, rather than each of its structural components, contains one DSB, assessed by direct DNA end-labelling or by the presence of phospho-Ku70. This is supported by the good agreement between the predicted number of DSBs induced by the dose of IR employed in this work and the numbers of $\gamma \mathrm{H} 2 \mathrm{AX}$ clusters in control cells. $\gamma \mathrm{H} 2 \mathrm{AX}$ clusters are spatially distributed in the nuclear space according to a pattern that is dependent on the progression of DDR. Such pattern recapitulates the previously described repair kinetics, underlining an euchromatin-to-heterochromatin repair trend, which is likely dictated by the chromatin compaction state: chromatin regions that were already in an open state (for example, marked by H3K36me3) would be repaired earlier, while compact chromatin requires further structural remodelling before the DNA repair machinery could eventually exert its activity (Fig. 9g), For the latter, actual DNA decondensation, assessed as decrease of DAPI intensity, occurred while maintaining the main local histone modification (for example, H3K9me3), thus uncoupling DNA compaction from histone modifications. While chromatin relaxation seems to be dispensable for the DNA repair to occur at pericentromeric heterochromatin ${ }^{44}$, we propose that the uncoupling of chromatin modifications and the actual chromatin decondensation is crucial to reestablish the original chromatin structure once DNA repair is accomplished.

In our 3D-SIM images, $\gamma \mathrm{H} 2 \mathrm{AX}$ clusters presented a discontinuous phosphorylation pattern, with $\gamma \mathrm{H} 2 \mathrm{AX}$ and CTCF showing mutually exclusive signals, although the two were in close spatial proximity. However, not all $\gamma \mathrm{H} 2 \mathrm{AX}$ nano-foci presented proximal CTCF foci. The latter likely consist of more than one CTCF molecule, and their detection may be influenced by a variety of factors, such as the CTCF-binding site density, differences in the binding affinity ${ }^{45}$ of such sites and CTCF protein levels. It is tempting to speculate that the discontinuously phosphorylated pattern we observed is due to the presence of multiple CTCF molecules bound to their cognate consensus sequences but not resolvable by our imaging techniques. To discriminate between each individual chromatin loop bound by a pair of CTCF molecules, would demand single molecule sensitivity in situ 3D methods. Nonetheless, it is equally possible that other chromatin structure regulators (for example, cohesion complex ${ }^{12}$ ), histone turnover (for example, during DNA repair ${ }^{46}$ ) as well as biological processes such as transcription ${ }^{11}$ antagonizing $\gamma \mathrm{H} 2 \mathrm{AX}$ formation and/or spreading along the chromosome contribute to the discontinuously phosphorylated pattern.

Finally, we show that CTCF has a critical role in the formation and spatial clustering of $\gamma \mathrm{H} 2 \mathrm{AX}$ nano-foci. CTCF-depleted cells present less $\gamma \mathrm{H} 2 \mathrm{AX}$ nano-foci, which are smaller and contain less DNA than those we observed in mock-treated cells. As a consequence, the DDR is delayed and the repair capability is diminished, despite the efficient activation of the main signalling effectors involved in H2AX phosphorylation (for example, DNA-PKcs or ATM). This indicates that a structural organization impairment-caused by CTCF depletion-results in a poor DDR. On CTCF depletion, the frequency of interactions of CTCF molecules with one another is decreased, leading to a diminished loop formation and a more sparse (that is, non-clustered) distribution of $\gamma \mathrm{H} 2 \mathrm{AX}$ nano-foci (Fig. 9b-g). Overall, this scenario emphasizes the need for a (dynamically) regulated $3 \mathrm{D}$ organization of the chromatin, whereby the 3D spatial proximity of chromatin loops could boost the local processivity of the committed kinases and assure an efficient DDR. In such context, because the CTCF-knocked-down cells display similar numbers of $\gamma \mathrm{H} 2 \mathrm{AX}$ nano-foci to the number of nano-foci cluster in control cells, we propose that in the absence of CTCF, spreading of $\gamma \mathrm{H} 2 \mathrm{AX}$ is impaired and, thus, this mark is restricted to the vicinity of the DSBs, that is, within one nano-focus (Fig. 9g).

In conclusion, our study demonstrates that the decreased levels of a single structural factor (CTCF), accounting for the (dynamic) stability of chromatin, per se dramatically hinder $\gamma \mathrm{H} 2 \mathrm{AX}$ spreading. While it is likely that additional factors (for example, DNA and histone methylation readers) contribute to this process, namely at heterochromatic regions, we propose that CTCF functions as a regulator of the structural component of DDR, preserving a crucial (dynamic) 3D organization of the chromatin and, thus, enabling an efficient DDR.

\section{Methods}

Cell culture and irradiation. Cervical carcinoma HeLa cells (ATCC No. CCL-2) cells were used throughout the study. A single exposure to $10 \mathrm{~Gy} \mathrm{X}$-ray was applied $\left(250 \mathrm{kV}, 16 \mathrm{~mA}, 2.5 \mathrm{~Gy} \mathrm{~min}^{-1}\right.$ - GE Isovolt Titan) to induce DNA damage and trigger DDR. On exposure to IR, cells were incubated in a humidified environment, with $5 \% \mathrm{CO}_{2}$ at $37^{\circ} \mathrm{C}$ as indicated. Sham-irradiated control cells were included. C2C12 (ATCC No CRL-1772) cells were used for CRISPR-Cas9 experiments. HeLa and $\mathrm{C} 2 \mathrm{C} 12$ cells were cultured in DMEM $\left(4.5 \mathrm{gl}^{-1}\right.$ glucose, Biochrom AG) supplemented with $10 \%$ and $20 \%$ fetal calf serum (Biochrom AG), respectively. All media were supplemented with $2 \mathrm{mM}$ L-glutamine (Sigma), $100 \mathrm{U}$ per ml penicillin and $100 \mu \mathrm{g} \mathrm{ml}^{-1}$ streptomycin (Sigma). All cell lines were tested for mycoplasma and found free of contamination (MycoAlert, Lonza).

Growth curve and cell cycle distribution. Cells were seeded $24 \mathrm{~h}$ before exposure to IR. After IR, cells were incubated for indicated times, before trypsinization and count with a coulter counter, in triplicates. The remaining cells were then fixed in $2 \%$ formaldehyde, permeabilized for 8 min with $0.5 \%$ Triton X-100 in PBS, stained with DAPI $\left(1 \mu \mathrm{g} \mathrm{ml}^{-1}\right)$ and analysed at the flow cytometer Partec PAS III system (Partec) for cell cycle distribution. Data were analysed with FlowJo software (Tree Star, Inc.).

Apoptosis assay. To detect apoptosis, TUNEL assay was performed according to the manufacturer's instructions (Roche, \#11684795910) and a minimum of 1,000 cells was scored by microscopy in two independent experiments.

Spectral karyotyping. Cells were treated with colcemid $\left(0.1 \mu \mathrm{g} \mathrm{ml}^{-1}\right.$; Invitrogen, Darmstadt, Germany) $2 \mathrm{~h}$ before collecting to accumulate metaphase cells.

\footnotetext{
Figure 9 | CTCF depletion inhibits $\gamma \mathbf{H} 2 \mathrm{AX}$ nano-foci and cluster formation and diminishes the DNA repair capability. (a) Number of CTCF foci in esiRNA-depleted cells before and during DDR. Black dots: median number of CTCF foci in wild-type cells. (b) Impairment of $\gamma \mathrm{H} 2 \mathrm{AX}$ nano-foci and 3Dclusters formation during DDR as assessed by immunofluorescence of 3D-SIM images in CTCF-depleted cells. Scale bar, $5 \mu \mathrm{m}$. (c) $\gamma \mathrm{H} 2 \mathrm{AX}$ nano-foci number distributions before and after IR, in CTCF siRNA-treated cells. Black dots: median number of $\gamma \mathrm{H} 2 \mathrm{AX}$ nano-foci of untreated cells (from Fig. 1).NS: two-tailed t-test, $P>0.05$. (d) $\gamma \mathrm{H} 2 \mathrm{AX}$ nano-foci DNA content distributions before and after IR, in CTCF siRNA-treated cells. Black dots: median DNA content of $\gamma \mathrm{H} 2 \mathrm{AX}$ nano-foci of untreated cells (from Fig. 2). (e) DNA fragmentation measured by the neutral comet assay. Boxes represent the mean of medians from four replicates (two biological replicates in duplicate), each consisting of 60 comet measurements. NS: not significant ( $t$-test, $P>0.05$ ). (f) $\gamma \mathrm{H} 2 \mathrm{AX}$ cluster distributions before and after IR, in CTCF siRNA-treated cells. Black dots: median number of $\gamma \mathrm{H} 2 \mathrm{AX}$ clusters in untreated cells (from Fig. 6 ). All boxes and whiskers are as in Fig. 1. Comparisons between time points (one-way ANOVA with Dunnett's correction) or between esiRNA-treated and wild-type cells (Wilcoxon/Mann-Whitney rank sum) are all statistically significant unless otherwise specified. (g) Model for cluster special arrangement during DDR, showing the time-dependent euchromatin-to-heterochromatin repair trend (top) and how $\gamma \mathrm{H} 2 \mathrm{AX}$ spreading is hampered by CTCF depletion with the concomitant loss of 3D-arrangement of chromatin loops (bottom).
} 
Chromosome preparations were made according to standard procedures and hybridized with the 24XCyte Multicolor FISH Probe Kit (MetaSystems, Altlussheim, Germany). Metaphase spreads were examined with an Axio Image Z1 microscope (Zeiss, Oberkochen, Germany) equipped with appropriate filter sets. At least 100 images of metaphases were taken, further processed using ISIS software (MetaSystems) and analysed to produce the karyotype.

CTCF knockdown. A number of $10^{5}$ cells were transfected with $15 \mathrm{nM}$ of a esiRNA pool (Sigma-Aldrich) using HiPerfect (Qiagen). The CTCF esiRNA is corresponding to the region 692-1195 of the human CTCF transcript (NM_006565.3). For mock treatments, cells were transfected using an esiRNA pool (Sigma-Aldrich) targeting the GFP gene. Cells were incubated $24-96 \mathrm{~h}$ post transfection and knockdown efficiency was monitored every $24 \mathrm{~h}$.

Immunoblotting. Whole-cell extracts were prepared by freeze and thaw lysis (three cycles) in $600 \mathrm{mM} \mathrm{NaCl}, 20 \mathrm{mM}$ Tris- $\mathrm{HCl}$ pH 7.8, 20\% glycerol. After SDS-PAGE, proteins were transferred onto PVDF membrane in semi-dry conditions. The membrane was then blocked in $5 \%$ non-fat dry milk buffer and incubated with mouse anti- $\gamma \mathrm{H} 2 \mathrm{AX}$ (Clone JBW301, Upstate, 1:5,000). Immunoblots were stained with corresponding HRP-conjugated secondary antibodies (GE Healthcare, 1:20,000) and detected with the enhanced chemiluminescence detection system (Amersham Biosciences). Quantification was performed using ImageJ.

For the validation of antibody specificity and cross-reactivity, a dilution series of synthetic peptides (CKATQASQEY; Peptide Specialty Laboratories $\mathrm{GmbH}$ ), with the underlined serine in either phosphorylated or non-phosphorylated form, was immobilized on a nitrocellulose membrane at the indicated concentrations and probed with anti- $\gamma \mathrm{H} 2 \mathrm{AX}$ and anti-H2AX as described above.

CTCF knockdown western blots were developed using a rabbit anti-CTCF (\#D31H2, Cell Signaling, 1:700) and a mouse anti-actin (AC-40, Sigma-Aldrich, $1: 1,000$ ) and overnight incubation at $4{ }^{\circ} \mathrm{C}$, followed by a direct immunofluorescence detection using anti-rabbit-IgG-Cy5 (\#711-175-152, Jackson, 1:1,000) and an anti-mouse-IgG-Alexa488 (A11029, Invitrogen, 1:1,000). Images were recorded using a AI600 Imager (Amersham) and quantified using ImageJ.

Immunofluorescence. Cells were fixed in $3.7 \%$ formaldehyde and permeabilized in $0.5 \%$ Triton X-100 in PBS at room temperature (RT). The following primary antibodies were used: mouse anti- $\gamma \mathrm{H} 2 \mathrm{AX}$ (Clone JBW301, 1:500, Upstate), rabbit anti-H3K9me3 (\#07-422, Upstate, 1:500), rabbit anti-H3K9me3 (\#39161, Active Motif, 1:500), rabbit anti-H3K36me3 (ab9050, Abcam, 1:2,000); rabbit anti-phospho-Ku70 (pS5) (\#ab61783, Abcam, 1:400); mouse anti-phospho-ATM (pS1981) (\#MAB3806, Millipore, 1:100); rabbit anti-phospho-DNA-PKcs (pS2056) (\#ab18192, Abcam, 1:100) and rabbit anti-CTCF (\#2899, Cell Signaling, 1:900). For phospho-Ku70 detection cells were prefixed in $1 \%$ formaldehyde and then extracted with $0.7 \%$ Triton X-100 two times by $5 \mathrm{~min}^{47}$ and subsequently fixed in $3.7 \%$ formaldehyde. Antibody incubation was performed at $4{ }^{\circ} \mathrm{C}$ over night in $1 \%$ BSA in PBS. For CLSM and 3D-SIM, signals were detected with goat anti-mouse-IgG-AlexaFluor 488, goat anti-rabbit-IgG-AlexaFluor 594 (1:800, Invitrogen), donkey anti-mouse-IgG-AlexaFluor 488 (A-21202, Thermo Fisher Scientific, 1:400), donkey anti-rabbit-IgG-AlexaFluor 594 (A-21207, Thermo Fisher Scientific, 1:400). For STED, $\gamma \mathrm{H} 2 \mathrm{AX}$ was detected with goat anti-mouse-IgG STAR 635P (\#2-0002-007-5, Abberior, 1:100) or goat anti-mouse-IgG STAR 580 (\#2-0002-005-1, Abberior, 1:100). DNA was counterstained with $36 \mathrm{nM}$ DAPI (for 3D-SIM), $1 \mu \mathrm{M}$ propidium iodide (confocal microscopy) or $2.5 \mu \mathrm{M}$ SiR-DNA (Spirochrome), before cells were mounted with Vectashield antifade medium (Vectorlabs).

CRISPR-Cas9 targeting to heterochromatic major satellite DNA. Subconfluent C2C12 cells were transfected with Cas9 (pCMV-hCas9, Addgene ID: 41815) and major satellite gRNAs (U6-MaSgRNA) by means of Lipofectamine 3000 (Thermo Fisher Scientific) according to the manufacturer's instructions. Cells were then fixed in $3.7 \%$ formaldehyde for $10 \mathrm{~min}$ and immunofluorescence followed (as described above).

DNA DSB detection by TUNEL assay. Cells were grown and irradiated as described above. At the indicated time points, cells were fixed in $3.7 \%$ paraformaldehyde for $10 \mathrm{~min}$. The fixation was quenched with $125 \mathrm{mM}$ glycine in PBS for $10 \mathrm{~min}$. Fixed cells where permeabilized in $0.5 \%$ Triton X-100 for $20 \mathrm{~min}$, and equilibrated for $10 \mathrm{~min}$ in blunting buffer $(100 \mathrm{mM}$ Tris- $\mathrm{HCl}, 50 \mathrm{mM} \mathrm{NaCl}$, $10 \mathrm{mM} \mathrm{MgCl}_{2}, 0.025 \%$ Triton X-100 and $5 \mathrm{mM}$ DTT, $\mathrm{pH} 7.5$ ). End repair was performed using $4 \mu \mathrm{l} \mathrm{T} 4$ polymerase (NEB: M0203S 3,000 units $\mathrm{ml}^{-1}$ ) and $4 \mu \mathrm{l} \mathrm{T4}$ polynucleotide kinase (NEB: M0201S 10,000 units $\mathrm{ml}^{-1}$ ) in $82 \mu \mathrm{l}$ blunting buffer, supplemented with $10 \mu \mathrm{l} 1 \mathrm{mM}$ dNTPs for $45 \mathrm{~min}$. Slides were then equilibrated in TdT buffer for $10 \mathrm{~min}$ and the TUNEL reaction was performed according to the 'In Situ Cell Death Detection Kit' (Roche) with Fluorescein modified dUTPs, for $4 \mathrm{~h}$ at $37^{\circ} \mathrm{C}$ according to the manufacturer's instructions. Following the TUNEL reaction, cells were blocked in 1\% BSA in PBS for 20 min. $\gamma \mathrm{H} 2 \mathrm{AX}$ staining was performed as described above. Incorporated fluorescein-dUTPs were detected by a rabbit anti-FITC (CUSABIO, 1:500) and a anti-rabbit-IgG Alexa488 secondary antibody (Jackson ImmunoResearch, 1:800). All steps were conducted at RT, unless otherwise specified.

Comet assay. DNA repair kinetics in CTCF knockdown cells were measured using the neutral comet assay. In brief, CTCF was depleted as described above and $72 \mathrm{~h}$ post esiRNA transfection, the cells were exposed to $10 \mathrm{~Gy} \mathrm{X}$-ray. At the indicated time points, cells were trypsinized and $2 \times 10^{5}$ cells ml $^{-1}$ were embedded in $0.8 \%$ low-melting point agarose (Sigma type VII). Lysis was performed for $4 \mathrm{~h}$ at $4^{\circ} \mathrm{C}$ in lysis buffer $(10 \mathrm{mM}$ Tris, $150 \mathrm{mM} \mathrm{NaCl}, 1 \% \mathrm{~N}$-laurylsarcosinate, $1 \%$ Triton X-100, 0.5\% DMSO, pH 8.0) and electrophoresis was done in $1 \times \mathrm{TBE}$ at $4^{\circ} \mathrm{C}\left(1 \mathrm{~V} \mathrm{~cm}^{-1}\right)$ for $25 \mathrm{~min}$. Slides were then dehydrated in $70 \%$ ethanol and rehydrated in staining buffer (TBE supplemented with SybrGreen, $1: 10,000)$ to stain the $\mathrm{DNA}^{48}$. Two biological replicates (in duplicates) were performed and 60 comets per slide were scored using Komet 4 (Kinetic Imaging Ltd.).

Microscopy. Confocal microscopy images were acquired using a Spinning Disk microscope (Perkin Elmer Vox1000) equipped with a $\times 60$ NA 1.4 oil immersion lens (CFI Apochromat TIRF), with a pixel size of $120 \mathrm{~nm}$ or with a Leica TCS SP5 confocal microscope using a Plan Apo $\times 63$ NA 1.4 oil immersion objective. Cells were recorded as $\mathrm{z}$-stacks with a $\mathrm{z}$-spacing of $0.2 \mu \mathrm{m}$.

Super-resolution microscopy images were acquired using a 3D structured illumination microscope (DeltaVision OMX V3, GE Healthcare) and a 2C STED 775 QUAD Scan microscope (Abberior Instruments). 3D-SIM was performed with a $\times 100$ NA 1.4 objective lens with a pixel size of $39 \mathrm{~nm}$ and a z-spacing of $125 \mathrm{~nm}$ (ref. 18). STED was performed with a $\times 100$ NA 1.4 Olympus UPlanSApo objective lens with a pixel size of $20 \mathrm{~nm}$ and excitation lasers of 488,594 or $640 \mathrm{~nm}$, and a $775 \mathrm{~nm}$ depletion laser.

High-content imaging was performed using the Operetta system (Perkin Elmer). Samples were imaged using a $\times 20$ NA 0.45 air objective with three planes of $1 \mu \mathrm{m}$ spacing, using the following filters: DAPI: excitation wavelength (ex): 360-400 nm, emission wavelength (em): 420-480 nm; Alexa488: ex: $460-490 \mathrm{~nm}$, em: 500-550 nm; Alexa594: ex: 560-580 nm, em: 590-640 nm.

Image analysis. For confocal microscopy, the images were analysed in Image using the nuclear staining as a mask to measure the total intensity of the $\gamma \mathrm{H} 2 \mathrm{AX}$ signal per nucleus. Foci were scored in 3D using Volocity (Perkin Elmer) by the following workflow: find objects (nucleus), threshold automatic, size minimum $500 \mathrm{~m}^{3}$; find foci: threshold 4,000 constant for pseudo-wide-field and 5,000 for deconvolved images, respectively. Minimum size: $0.05 \mu \mathrm{m}^{3}$, followed by 'separate touching objects' with a guide size of $0.5 \mu \mathrm{m}^{3}$. Different thresholds were applied, because pseudo-wide-field and deconvolved images are in different bit depth. All counts were double-checked by manual counting of randomly chosen samples by at least three experimenters.

For CRISPR-Cas9 experiments, confocal images of $\mathrm{C} 2 \mathrm{C} 12$ cells were segmented into background, nuclei and chromocentres by pixel-wise classification via supervised machine learning (default Random Forest classifier and pixel features from the Trainable Weka Segmentation plugin in Fiji). The classifier was trained on manually labelled pixels of the DAPI channel in one image and then applied to all images. For each image, mean intensities in the $\mathrm{H} 3 \mathrm{~K} 9 \mathrm{me} 3$ and $\gamma \mathrm{H} 2 \mathrm{AX}$ channels were determined for each chromocentre object $\left(>100 \mathrm{px}^{2}\right)$ within the largest object in the nuclear mask. To analyse DNA decondensation at repair sites in CRISPR-Cas9 experiments STED images of $\mathrm{C} 2 \mathrm{C} 12$ cells were segmented into background, nuclei and chromocentres by pixel-wise classification as described above for confocal images. The classifier was trained on manually labelled pixels of the SiR-DNA channel in one image and then applied to all images (each image's pixel intensity range was mapped to the 8-bit range to account for differences in staining intensities). For each image, the circularity of chromocentre objects $\left(>100 \mathrm{px}^{2}\right)$ within the nucleus was determined. Three rounds of binary erosion with a $3 \times 3$ px-box followed by three rounds of binary dilation were applied to the segmentation results to smooth the borders of segmented objects.

3D-SIM images were exported from the DeltaVision software (softWoRx 6.0 Beta 19, Applied Precision) and converted to 16-bit images per channel. Foci counting was done using Volocity 6.3 (Perkin Elmer) or with the 3D foci picker plugin in ImageJ (imagej.nih.gov/ij/). Nearly identical results were obtained and the numbers from Volocity were used. In detail, the individual z-sections were imported and merged to a volume with the above-mentioned pixel sizes and $\mathrm{z}$-spacing. First, the nucleus was identified by setting a manual threshold and a lower volume limit of $200 \mu^{3}$ followed by a 'Fill in Holes' step and two iterations of 'Dilate' and 'Close' to fill in all the DAPI weak volumes. The intensities and voxel coordinates of the whole nucleus were registered. Next, the $\gamma \mathrm{H} 2 \mathrm{AX}$ and H3K36me3 or $\mathrm{H} 3 \mathrm{~K} 9 \mathrm{me} 3$ foci were identified with a lower threshold of 1,000 and a minimum object size of $0.001 \mu \mathrm{m}^{3}$. To separate close spaced objects, a final 'Separate Touching Objects' step with a nominal volume of $0.05 \mu \mathrm{m}^{3}$ was used. The foci identified were restricted to the previously defined nuclear volume to remove possible unspecific signals from outside of the nucleus.

3D-SIM pseudo-wide-field imaging: after sample acquisition, the pseudo-widefield images were calculated using softWoRx 6.0 Beta 19 according to the following 
workflow: the raw data from each 3D-SIM image $\mathrm{z}$-stack was subdivided to isolate the first angle of acquisition. To this purpose, the maximum number of $z$-sections in each individual stack is divided by three. Then the projected five grid shifted section is averaged per z-position and colour channel. After that, the voxel dimensions are adjusted from 0.625 to $0.125 \mu \mathrm{m}$ in the z-dimension by adjusting the file headers. The alignment of the new stack was done with the parameters used for 3D-SIM reconstruction. The following parameters of the softWoRx software were used: normalize intensity, use photosensor, correct bleaching, replace z-lines and smooth z-lines. To reverse the optical distortion in the images, the aligned 3D stack was deconvolved with the instrument-specific optical transfer function (OTF) with the following settings: 'enhanced ratio (aggressive)' and 'noise filtering medium'.

For CTCF distance analysis, the previously described protocol was extended as follows: CTCF domains detection was restricted to the nuclear volume, with an automated threshold and a minimum size of $0.001 \mu \mathrm{m}^{3}$. Then, the segmented $\gamma \mathrm{H} 2 \mathrm{AX}$ nano-foci were extended in all dimensions by three voxels $(117 \times 117 \times 375 \mathrm{~nm})$ and the resulting $\gamma \mathrm{H} 2 \mathrm{AX}$ nano-foci volume was subtracted to obtain the $\gamma \mathrm{H} 2 \mathrm{AX}$ foci shells. Finally, the Euclidian distances between each $\gamma \mathrm{H} 2 \mathrm{AX}$ nano-focus and the closest CTCF domain were measured. All identified foci with the corresponding $3 \mathrm{D}$ coordinates and intensities for all recorded channels were exported and post-processed in $\mathrm{R}^{49}$. ImageJ and UCSF chimera ${ }^{50}$ were used for image visualization and $3 \mathrm{D}$ rendering, respectively. Simulations of CTCF and $\gamma \mathrm{H} 2 \mathrm{AX}$ distributions were run under R, using rgl and sphereplot packages. Hundred simulations of a sphere matching the average nuclear size of cells were run per time point. Every simulation contained objects whose numbers matched CTCF and $\gamma \mathrm{H} 2 \mathrm{AX}$ foci we recorded in 3D-SIM images.

For STED images, object dimensions (for example, diameters) were measured by manual object segmentation of randomly selected foci in ImageJ, using the analyse particle tool. For high-content images, analysis was performed using Harmony software (Perkin Elmer) with the following workflow: maximum projection of the planes, flatfield correction, find nuclei in DAPI channel, method $\mathrm{M}$, splitting coefficient 0.1 , general threshold 0.4 and guide size of $15 \mu \mathrm{m}$ in diameter. Calculate intensity and morphology parameters for the nuclei. Discard nuclei touching the border, smaller than $100 \mu \mathrm{m}^{2}$ and larger than $350 \mu \mathrm{m}^{2}$. Filter nuclei for roundness $>0.83$ and with a 4 px Haralick contrast $>0.8$ and a DAPI signal CV of less than $30 \%$. Measure the mean and integrated intensity for DAPI, $\gamma \mathrm{H} 2 \mathrm{AX}$ and CTCF in the selected nuclei areas.

ChIP. Cells were fixed with $1 \%$ formaldehyde for $10 \mathrm{~min}$ at RT and cross-link was quenched with $125 \mathrm{mM}$ glycine (5 min at RT). Nuclei were isolated after mild lysis in hypotonic buffer (10 mM HEPES pH $8,1.5 \mathrm{mM} \mathrm{MgCl}_{2}$, $60 \mathrm{mM} \mathrm{KCl}$ ) and 20 strokes in a tight dounce homogenizer. Chromatin was sheared in sonication buffer (0.5\% SDS, $10 \mathrm{mM}$ EDTA, $50 \mathrm{mM}$ Tris-HCl $\mathrm{pH}$ 8.1). Fragmentation of chromatin was carried out by ultrasound treatment (Bioruptor UCD200) so that fragments of 200-300 bp length were obtained. Chromatin from $1 \times 10^{6}-2 \times 10^{6}$ cells was immunoprecipitated with anti- $\gamma \mathrm{H} 2 \mathrm{AX}$ (Clone JBW301, Upstate, $3 \mu \mathrm{g}$ ) antibody. Chromatin was then incubated $\mathrm{ON}$ at $4{ }^{\circ} \mathrm{C}$ with protein G-coated magnetic beads (ChIP-IT Express, Active Motif). The collected chromatin (ChIP sample) was then reverse-crosslinked in the presence of $200 \mathrm{mM} \mathrm{NaCl}$ at $65^{\circ} \mathrm{C}$ for at least $5 \mathrm{~h}$, followed by RNase $\mathrm{A}\left(50 \mu \mathrm{g} \mathrm{m}{ }^{-1}\right)$ treatment for $30 \mathrm{~min}$ at $37^{\circ} \mathrm{C}$ and proteinase $\mathrm{K}\left(100 \mathrm{\mu g} \mathrm{ml}^{-1}\right)$ treatment for $3 \mathrm{~h}$ at $50^{\circ} \mathrm{C}$. DNA elution was carried out in $1 \% \mathrm{SDS}, 100 \mathrm{mM} \mathrm{NaHCO}_{3}$, in a rotary shaker at RT for $15 \mathrm{~min}$. Pure DNA was isolated using the Qiagen PCR purification kit and 15-30 ng of size selected DNA fragments (Qubit fluorometric quantification) were used to produce ChIP-seq libraries (Illumina ChIP-Seq DNA sample Prep Kit). Input sample was essentially prepared following the same protocol, but the immunoprecipitation step was skipped.

\section{Next-generation sequencing and data analyses. ChIP-Seq libraries were} processed through a high-throughput sequencing pipeline (Illumina Genome Analyzer II). Reads were mapped to the human genome (University of California, Santa Cruz (UCSC) hg19 assembly, based on the National Center for Biotechnology Information (NCBI) build 37.1) by means of SOAP2 software ${ }^{51}$, allowing up to two mismatches for each $36 \mathrm{bp}$ read. All data sets were deposited in the Gene Expression Omnibus database (accession number: GSE60526). All $\gamma \mathrm{H} 2 \mathrm{AX}$ ChIP-Seq tracks were smoothed with a moving average of five intervals before further analysis. Genomic features and correlation analysis: all genomic features data were retrieved from publicly available databases (UCSC) (Supplementary Table 3). Most of the data were generated in HepG2 cells, but not all. Data that were originally generated in the hg18 assembly were transposed to hg19 using LiftOver (http://genome.ucsc.edu/cgi-bin/hgLiftOver). Reads per kilobase per million reads (RPKM) ${ }^{52}$ were calculated for non-overlapping $10 \mathrm{~kb}$ genomic intervals for all sequence tracks. The features were further normalized to the corresponding genome-wide average and correlation with $\gamma \mathrm{H} 2 \mathrm{AX}$ tracks was performed (Spearman's $\rho$ correlation coefficient with $P<2.2 \times 10^{-16}$ in all cases).

Statistical analysis. Overall, sample size was chosen so that groups (for example, time points) had comparable numbers (for example, number of imaged cells), whenever possible. High-content microscopy and next-generation sequencing provided large data sets ensuring statistical significance. All statistical analysis has been performed using R or GraphPad Prism. Briefly, in case data were normally distributed (Shapiro-Wilk test), ANOVA or Student's $t$-test were performed for groups or pairs, respectively. Else, Kruskal-Wallis or Wilcoxon/Mann-Whitney rank sum tests were used for groups or pairs, respectively.

Integration of 3D-SIM and ChIP-Seq data. To integrate the ChIP-Seq data with 3D-SIM information, we first generated 25 independent profiles by applying a smoothing factor to each $\gamma$ H2AX ChIP-Seq data set (Supplementary Fig. 4A). Such smoothing factor is a moving average ranging from 1 (no smoothing) to 25 genomic intervals (indicated as '1D', in Supplementary Fig. 4A). In parallel, we measured the volume fraction occupied by $\gamma \mathrm{H} 2 \mathrm{AX}$ nano-foci as well as their corresponding DNA content, before and during the DDR (Supplementary Fig. 4B). In response to ionizing radiation, we observed an increase of the mean $\gamma \mathrm{H} 2 \mathrm{AX}$-occupied nuclear volume (from $0.21 \pm 0.21 \%$ to $7.81 \pm 3.19 \%$ ), which recapitulated the DDR (the volume was reduced to $3.70 \pm 1.39 \%$ and $0.66 \pm 0.43 \%$, at $3 \mathrm{~h}$ or $24 \mathrm{~h}$ post-ionizing radiation, respectively). Next, we applied the mean volume fractions $(0.21 \%, 7.81 \%, 3.70 \%$ and $0.66 \%$ for unirradiated, $0.5 \mathrm{~h}, 3 \mathrm{~h}$ and $24 \mathrm{~h}$, respectively) to filter the previously smoothed genomic $\gamma \mathrm{H} 2 \mathrm{AX}$ ChIP-Seq data so that only the $10 \mathrm{~kb}$ genomic intervals from the top percentiles of the read density distributions were retrieved (Supplementary Fig. 4C). For example, as for the unirradiated cells, we sampled the 99.79th percentile (top 100-0.21\%) of the intervals, while for the $0.5 \mathrm{~h}$ time point, we sampled the 92.19th percentile (top $100-7.81 \%$ ) of the total RPKM $\gamma \mathrm{H} 2 \mathrm{AX}$ ChIP-Seq distribution. A representative image of filtered ChIP-Seq profiles is shown in Supplementary Fig. 4D. By applying these imaging-based thresholds, we obtained a linear coverage of $4.7 \mathrm{Mbp}$, 159.0 Mbp, $92.3 \mathrm{Mbp}$ and $21.8 \mathrm{Mbp}$, at unirradiated, $0.5 \mathrm{~h}, 3 \mathrm{~h}$ and $24 \mathrm{~h}$ time points, respectively (Supplementary Fig. 4E). Finally, we employed the numbers of 3D $\gamma \mathrm{H} 2 \mathrm{AX}$ nano-foci to match the numbers of $1 \mathrm{D}$ nano-domains as follows: first, the number of 3D $\gamma \mathrm{H} 2 \mathrm{AX}$ nano-foci before and after the DDR was scaled down to the haploid genome size to match the genomic data (ploidy correction factor: HeLa genome size $_{\text {haploid reference }}$ genome size $=3.12$ ); next, we chose the smoothing factor at which the number of $\gamma \mathrm{H} 2 \mathrm{AX}$ nano-foci and the number of retrieved genomic intervals matched best, at any given time point (Supplementary Fig. $4 \mathrm{~A}$, over-imposed crosses). All ChIP-Seq domains identified via such approach are referred to as ' $1 \mathrm{D}$ domains' and an estimate of the $1 \mathrm{D}$ domain size distribution is presented in Supplementary Fig. 4F.

Data availability. Next-generation sequencing results are available at GEO (https://www.ncbi.nlm.nih.gov/geo/) under the accession number GSE60526. Other data that support the findings of this study are available from the corresponding author on reasonable request.

\section{References}

1. Rogakou, E. P., Pilch, D. R., Orr, A. H., Ivanova, V. S. \& Bonner, W. M. DNA double-stranded breaks induce histone H2AX phosphorylation on serine 139. J. Biol. Chem. 273, 5858-5868 (1998).

2. Stucki, M. \& Jackson, S. P. GammaH2AX and MDC1: anchoring the DNA-damage-response machinery to broken chromosomes. DNA Repair 5, 534-543 (2006).

3. Bartkova, J. et al. DNA damage response as a candidate anti-cancer barrier in early human tumorigenesis. Nature 434, 864-870 (2005).

4. Gorgoulis, V. G. et al. Activation of the DNA damage checkpoint and genomic instability in human precancerous lesions. Nature 434, 907-913 (2005).

5. Turinetto, V. \& Giachino, C. Multiple facets of histone variant H2AX: a DNA double-strand-break marker with several biological functions. Nucleic Acids Res. 43, 2489-2498 (2015).

6. Berkovich, E., Monnat, Jr R. J. \& Kastan, M. B. Roles of ATM and NBS1 in chromatin structure modulation and DNA double-strand break repair. Nat. Cell Biol. 9, 683-690 (2007).

7. Meier, A. et al. Spreading of mammalian DNA-damage response factors studied by ChIP-chip at damaged telomeres. EMBO J. 26, 2707-2718 (2007).

8. Savic, V., Sanborn, K. B., Orange, J. S. \& Bassing, C. H. Chipping away at gamma-H2AX foci. Cell Cycle 8, 3285-3290 (2009).

9. Kim, J. A., Kruhlak, M., Dotiwala, F., Nussenzweig, A. \& Haber, J. E. Heterochromatin is refractory to gamma-H2AX modification in yeast and mammals. J. Cell Biol. 178, 209-218 (2007).

10. Shroff, R. et al. Distribution and dynamics of chromatin modification induced by a defined DNA double-strand break. Curr. Biol. 14, 1703-1711 (2004).

11. Iacovoni, J. S. et al. High-resolution profiling of gammaH2AX around DNA double strand breaks in the mammalian genome. EMBO J. 29, 1446-1457 (2010).

12. Caron, P. et al. Cohesin protects genes against gammaH2AX Induced by DNA double-strand breaks. PLoS Genet. 8, e1002460 (2012).

13. Ong, C. T. \& Corces, V. G. CTCF: an architectural protein bridging genome topology and function. Nat. Rev. Genet. 15, 234-246 (2014).

14. Dixon, J. R. et al. Topological domains in mammalian genomes identified by analysis of chromatin interactions. Nature 485, 376-380 (2012). 
15. Rao, S. S. et al. A 3D map of the human genome at kilobase resolution reveals principles of chromatin looping. Cell 159, 1665-1680 (2014).

16. Gonzalez-Sandoval, A. et al. Perinuclear anchoring of H3K9-methylated chromatin stabilizes induced cell fate in C. elegans embryos. Cell 163, 1333-1347 (2015).

17. Pope, B. D. et al. Topologically associating domains are stable units of replication-timing regulation. Nature 515, 402-405 (2014).

18. Schermelleh, L., Heintzmann, R. \& Leonhardt, H. A guide to super-resolution fluorescence microscopy. J. Cell Biol. 190, 165-175 (2010).

19. Hell, S. W. \& Wichmann, J. Breaking the diffraction resolution limit by stimulated emission: stimulated-emission-depletion fluorescence microscopy. Opt. Lett. 19, 780-782 (1994).

20. Lobrich, M. et al. GammaH2AX foci analysis for monitoring DNA doublestrand break repair: strengths, limitations and optimization. Cell Cycle 9, 662-669 (2010).

21. Halazonetis, T. D., Gorgoulis, V. G. \& Bartek, J. An oncogene-induced DNA damage model for cancer development. Science 319, 1352-1355 (2008).

22. Tsantoulis, P. K. et al. Oncogene-induced replication stress preferentially targets common fragile sites in preneoplastic lesions. A genome-wide study. Oncogene 27, 3256-3264 (2008).

23. Lee, C. S., Lee, K., Legube, G. \& Haber, J. E. Dynamics of yeast histone H2A and H2B phosphorylation in response to a double-strand break. Nat. Struct. Mol. Biol. 21, 103-109 (2014).

24. Kolasinska-Zwierz, P. et al. Differential chromatin marking of introns and expressed exons by H3K36me3. Nat. Genet. 41, 376-381 (2009).

25. Peters, A. H. et al. Histone $\mathrm{H} 3$ lysine 9 methylation is an epigenetic imprint of facultative heterochromatin. Nat. Genet. 30, 77-80 (2002).

26. Chiolo, I. et al. Double-strand breaks in heterochromatin move outside of a dynamic HP1a domain to complete recombinational repair. Cell 144, 732-744 (2011).

27. Jakob, B. et al. DNA double-strand breaks in heterochromatin elicit fast repair protein recruitment, histone $\mathrm{H} 2 \mathrm{AX}$ phosphorylation and relocation to euchromatin. Nucleic Acids Res. 39, 6489-6499 (2011).

28. Boettiger, A. N. et al. Super-resolution imaging reveals distinct chromatin folding for different epigenetic states. Nature 529, 418-422 (2016).

29. Meyer, B. et al. Clustered DNA damage induces pan-nuclear H2AX phosphorylation mediated by ATM and DNA-PK. Nucleic Acids Res. 41, 6109-6118 (2013).

30. Rothkamm, K., Kruger, I., Thompson, L. H. \& Lobrich, M. Pathways of DNA double-strand break repair during the mammalian cell cycle. Mol. Cell Biol. 23, 5706-5715 (2003).

31. Polo, S. E. \& Jackson, S. P. Dynamics of DNA damage response proteins at DNA breaks: a focus on protein modifications. Genes Dev. 25, 409-433 (2011).

32. Newman, H. C., Prise, K. M., Folkard, M. \& Michael, B. D. DNA double-strand break distributions in X-ray and alpha-particle irradiated V79 cells: evidence for non-random breakage. Int. J. Radiat. Biol. 71, 347-363 (1997).

33. Lobrich, M., Rydberg, B. \& Cooper, P. K. Repair of x-ray-induced DNA double-strand breaks in specific Not I restriction fragments in human fibroblasts: joining of correct and incorrect ends. Proc. Natl Acad. Sci. USA 92, 12050-12054 (1995).

34. Chaumeil, J. \& Skok, J. A. The role of CTCF in regulating V(D)J recombination. Curr. Opin. Immunol. 24, 153-159 (2012).

35. Cuddapah, S. et al. Global analysis of the insulator binding protein CTCF in chromatin barrier regions reveals demarcation of active and repressive domains. Genome Res. 19, 24-32 (2009).

36. Hou, C., Zhao, H., Tanimoto, K. \& Dean, A. CTCF-dependent enhancerblocking by alternative chromatin loop formation. Proc. Natl Acad. Sci. USA 105, 20398-20403 (2008).

37. Fu, Y., Sinha, M., Peterson, C. L. \& Weng, Z. The insulator binding protein CTCF positions 20 nucleosomes around its binding sites across the human genome. PLoS Genet. 4, e1000138 (2008).

38. Phillips, J. E. \& Corces, V. G. CTCF: master weaver of the genome. Cell 137, 1194-1211 (2009).

39. Kim, T. H. et al. Analysis of the vertebrate insulator protein CTCF-binding sites in the human genome. Cell 128, 1231-1245 (2007).

40. Schmidt, D. et al. A CTCF-independent role for cohesin in tissue-specific transcription. Genome Res. 20, 578-588 (2010).

41. Tark-Dame, M., Jerabek, H., Manders, E. M., Heermann, D. W. \& van Driel, R. Depletion of the chromatin looping proteins CTCF and cohesin causes chromatin compaction: insight into chromatin folding by polymer modelling. PLoS Comput. Biol. 10, e1003877 (2014)

42. Solovei, I., Thanisch, K. \& Feodorova, Y. How to rule the nucleus: divide et impera. Curr. Opin. Cell Biol. 40, 47-59 (2016).
43. Lopez Perez, R. et al. Superresolution light microscopy shows nanostructure of carbon ion radiation-induced DNA double-strand break repair foci. FASEB J 30, 2767-2776 (2016).

44. Tsouroula, K. et al. Temporal and spatial uncoupling of DNA double strand break repair pathways within mammalian heterochromatin. Mol. Cell 63, 293-305 (2016).

45. Plasschaert, R. N. et al. CTCF binding site sequence differences are associated with unique regulatory and functional trends during embryonic stem cell differentiation. Nucleic Acids Res. 42, 774-789 (2014).

46. Ikura, T. et al. DNA damage-dependent acetylation and ubiquitination of H2AX enhances chromatin dynamics. Mol. Cell Biol. 27, 7028-7040 (2007).

47. Anton, T., Bultmann, S., Leonhardt, H. \& Markaki, Y. Visualization of specific DNA sequences in living mouse embryonic stem cells with a programmable fluorescent CRISPR/Cas system. Nucleus 5, 163-172 (2014).

48. Greinert, R. et al. UVA-induced DNA double-strand breaks result from the repair of clustered oxidative DNA damages. Nucleic Acids Res. 40, 10263-10273 (2012).

49. R-Core-Team. R. A language and environment for statistical computing (2014)

50. Pettersen, E. F. et al. UCSF chimera--a visualization system for exploratory research and analysis. J. Comput. Chem. 25, 1605-1612 (2004).

51. Li, R. et al. SOAP2: an improved ultrafast tool for short read alignment. Bioinformatics 25, 1966-1967 (2009).

52. Mortazavi, A., Williams, B. A., McCue, K., Schaeffer, L. \& Wold, B. Mapping and quantifying mammalian transcriptomes by RNA-Seq. Nat. Methods 5, 621-628 (2008)

\section{Acknowledgements}

We are indebted to Dr Diana Pignalosa and Dr Sylvia Ritter for SKY analysis. This work was supported by grants of the Deutsche Forschungsgemeinschaft (GRK1657/TP1B to M.C.C.; GRK1657/TP1C to A.R.; DFG CA198/8-1 and 2 to M.C.C.), the Bundesministerium für Bildung und Forschung Grants 02NUK017D and 02S8355 to M.C.C. and 02NUK036D to A.R. and the Beilsetein-Institute, NanoBiC collaboration.

\section{Author contributions}

F.N. and A.R. performed the ChIP-Seq experiments. A.M. and A.R. performed the 3D-SIM microscopy. A.S., H.H. and D.H. performed the STED microscopy. F.N., A.R. and W.Y. performed the bioinformatics. T.A. and D.H. performed the CRISPR-Cas 9 experiments. M.C.C., F.N. and A.R. designed the project, analysed the data and wrote the manuscript. M.D., W.C., H.L. and G.T.-S. provided tools and expertise, and revised the manuscript.

\section{Additional information}

Supplementary Information accompanies this paper at http://www.nature.com/ naturecommunications

Competing interests: The authors declare no competing financial interests.

Reprints and permission information is available online at http://npg.nature.com/ reprintsandpermissions/

How to cite this article: Natale, F. et al. Identification of the elementary structural units of the DNA damage response. Nat. Commun. 8, 15760 doi: 10.1038/ncomms15760 (2017).

Publisher's note: Springer Nature remains neutral with regard to jurisdictional claims in published maps and institutional affiliations.

Open Access This article is licensed under a Creative Commons Attribution 4.0 International License, which permits use, sharing, adaptation, distribution and reproduction in any medium or format, as long as you give appropriate credit to the original author(s) and the source, provide a link to the Creative Commons license, and indicate if changes were made. The images or other third party material in this article are included in the article's Creative Commons license, unless indicated otherwise in a credit line to the material. If material is not included in the article's Creative Commons license and your intended use is not permitted by statutory regulation or exceeds the permitted use, you will need to obtain permission directly from the copyright holder. To view a copy of this license, visit http://creativecommons.org/ licenses/by/4.0/

C) The Author(s) 2017 